Renewable Energy - An International Journal

Impact Factor: 4.90

ISSN: 0960-1481; Publisher: ELSEVIER

Accepted August $28^{\text {th }} 2018$

\title{
PERISTALTIC PUMPING OF MAGNETIC NANOFLUIDS WITH THERMAL RADIATION AND TEMPERATURE- DEPENDENT VISCOSITY EFFECTS: MODELLING A SOLAR MAGNETO-BIOMIMETIC NANOPUMP
}

\author{
${ }^{1}$ J. Prakash, ${ }^{2}$ E.P. Siva, ${ }^{\#}$ D Tripathi, ${ }^{4}$ S. Kuharat and ${ }^{4}$ O. Anwar Bég \\ ${ }^{1}$ Department of Mathematics, Agni College of Technology, Thalambur, Chennai - 600130, India. \\ ${ }^{2}$ Department of Mathematics, SRM Institute of Science and Technology, Kattankulathur - 603203, India. \\ ${ }^{3}$ Department of Mechanical Engineering, Manipal University Jaipur, Rajasthan-303007, India \\ ${ }^{4}$ Department of Aeronautical and Mechanical Engineering, Salford University, Manchester, M54WT, UK.
}

\#Corresponding author: dharmendra.tripathi@jaipur.manipal.edu

\begin{abstract}
Nanofluids have shown significant promise in the thermal enhancement of many industrial systems. They have been developed extensively in energy applications in recent years. Solar energy systems are one of the most promising renewables available to humanity and these are increasingly being re-designed to benefit from nanofluids. Most designs of solar collectors involve fixed (rigid) geometries which may be cylindrical, parabolic, tubular or flat-plate types. Modern developments in biomimetics have identified that deformable conduit structures may be beneficial for sustainable energy systems. Motivated by these aspects, in the current work we present a novel model for simulating a biomimetic peristaltic solar magnetohydrodynamic nanofluid-based pump. The working fluid is a magnetized nanofluid which comprises a base fluid containing suspended magnetic nano-particles. The novelty of the present work is the amalgamation of biomimetics (peristaltic propulsion), magnetohydrodynamics and nanofluid dynamics to produce a hybrid solar pump system model. Heat is transferred via distensibility of the conduit in the form of peristaltic thermal waves and buoyancy effects. An externally applied magnetic field achieves the necessary circuit design for generating Lorentzian magnetic body force in the fluid. A variable viscosity modification of the Buongiorno nanofluid model is employed which features thermophoretic body force and Brownian dynamic effects. To simulate solar loading conditions a thermal radiative flux model is also deployed. An asymmetric porous channel is investigated with multiple amplitudes and phases for the wall wavy motion. The channel also contains a homogenous, isotropic porous medium which is simulated with a modified Darcy model. Heat generation/absorption effects are also examined. The electrically-conducting nature of the nanofluid invokes magnetohydrodynamic effects. The moving boundary value problem is normalized and linearized using the lubrication approach. Analytical solutions are derived for axial velocity, temperature and nanoparticle volume fraction. Validation is conducted with Maple numerical quadrature. Furthermore, the salient features of pumping and trapping phenomena discourse briefly. The observations demonstrate promising features of the solar magnetohydrodynamic peristaltic nanofluid pump which may also be exploited in spacecraft applications, biological smart drug delivery etc.
\end{abstract}

KEYWORDS: Peristaltic solar pump; magnetohydrodynamics; nanofluids; porous medium; thermal radiation; perturbation analysis.

\section{INTRODUCTION}

Modern trends in renewable and sustainable energy systems have witnessed a proliferation of novel designs. Pre-eminent in such systems is the solar energy collector which exploits, the key 
energy source available to the earth, namely the sun. Pioneering studies of radiative transport in solar collectors and surface properties were led by Hottel at MIT from the 1930s to the 1960s and much of this work is summarized in an excellent monograph [1]. Since 1970 a worldwide effort has been underway to increase the durability, resilience and thermal efficiency of solar collector systems. Solar technology has subsequently infiltrated a vast array of devices including solar ponds [2], solar absorption chillers [3], solar towers [4], solar cooling systems [5], CHP refrigeration solar hybrid plants [6], solar-petrochemical steam control plants [7], green buildings (photovoltaic facades) [8] and solar automobiles [9]. Another important application of solar technology is the solar-powered pump [10] which has many uses including heating, waste transport, medical fermentation batch processing etc. A significant development in solar technology has also been the advent of nanofluids. Nanofluids were introduced by Choi [11] constitute a significant advance in fluid dynamics technology and are synthesized by doping conventional base fluids (e.g. water, mineral oil, air, etc.) with carefully designed nano-particles. The resulting suspension achieves improved thermal conductivity and modified viscosity properties. The surface area per unit volume of nanoparticles is much larger (millions of times) than that of conventional microparticles. The number of surface atoms per unit of interior atoms of nanoparticles is very large. These characteristics can be exploited in many complex systems including medical engineering, energy engineering and materials processing. Nanofluids have infiltrated into many areas of energy and also biomedical technology as they may be manipulated to yield more biologically friendly, sustainable and durable products. Khanafer and Vafai [12] presented a lucid summary of solar nanofluid device applications, emphasizing that efficiency of any solar thermal system is dictated by thermophysical properties (viscosity, density, thermal conductivity and specific heat) of the operating fluid and the geometric characteristics. Critical features of nanofluids for improving solar collector and pump efficiency are types of the nanoparticles (metallic based work best e.g. copper, silver, titanium), nanoparticles volumetric concentration in the base fluid and the nanofluid viscosity and conductivity. The inclusion of copper nanoparticles considerably elevates the heat gain capacity of a solar pump. Carbon nanotube nanofluids not only improve the efficiency of solar collectors but have the added advantage of decreasing $\mathrm{CO}_{2}$ emissions. It should also be noted that there are a diverse range of mathematical models available for simulating nanofluid transport phenomena which have also been addressed in Khanafer and Vafai [12]. These include the Buongiorno two-component model [13] which emphasizes thermophoretic forces and Brownian 
motion dynamics as the key contributors to thermal conductivity enhancement. The other popular model is Tiwari and Das model [14] which simulates the nanoscale effect based on volume fraction (concentration) of the nano-particles. This model prioritizes thermal conductivity and viscosity of the nanofluid by appropriate functions formulated in terms of the nanoparticle volume fraction. However, it is restricted only to a momentum and energy balance and does not feature a separate species concentration balance equation for the nano-particles. The Tiwari-Das model has the serious drawback of confining the nanoparticle contribution to volume fraction rather than via a separate species conservation equation. The Buongiorno model therefore while it ignores volume fraction effects does compensate for this by a discrete equation for the nano-particle concentration diffusion and is more comprehensive therefore for complex flow simulations where Brownian motion can be modelled. Many recent studies have been communicated on nanofluid solar pumps and collector systems including Shamshirgaran et al. [15] (on energy dynamics of copper nanofluid flat plate collectors), Owlabi et al. [16] (on integrated silver oxide doped nano-solar cells), Cingarapu et al. [17] (on tin-based nanofluids for solar pumping designs), Ravindran [18] (on nano-fuels for rocket propulsion and solar power), Abid et al. [19] (on salt-based ionic nanofluids for parabolic collectors) and Alashkar and Gadalla [20] (focused on gold and zinc oxide nanofluids for solar power pumps). All these studies confirmed the considerable elevation in thermal efficiency and sustainability of solar power designs attained with judicious deployment of metallic nano-particles.

Another important renewable pump design is the magnetohydrodynamic (MHD) pump [21, 22]. These pumps employ the Lorentz magnetic body force effect, based on the injection of an electric current into two electrodes located at sidewalls facing each other in a microchannel. This charge injection produces a significant transversal ionic current in the microchannel, which is simultaneously subjected to a magnetic field oriented at an angle of $90^{\circ}$ to the current direction and microchannel axis. Conventional MHD micropumps can generate only small values for pump rate and achievable pressure and the performance is strongly controlled by the ionic conductivity of the pumping fluid. In DC MHD pumps, in particular, electrolytic bubble generation at the injection electrodes can is a key problem [23-25]. However, recent studies with magnetized nanofluids have largely mitigated these and other issues and have simultaneously achieved the marked enhancement in pump efficiency and longevity. Important efforts in this regard include the work of Shahidian et al. [26] which explored the impact of different electrical conductivities 
of nano-particle doped ionic fluids on overall efficiency. Further studies emphasizing the promise of nanofluid-based MHD pumps includes Qian and Bau [27], Shahidian et al. [28] and Joo and Lee [29]. Another exciting development in renewable energy has been the amalgamation of solar pump technology with magnetohydrodynamics (MHD). Recent Work at RPI [30], has established that due to higher temperature generated, solar MHD pumps attain a demonstrably greater efficiency than standard solar thermal pump technologies which only operate at a much lower temperature. This new branch of renewables is termed concentrating solar MHD Power Utility and is currently being commercialized in the USA. It combines the benefits of high energy radiative power sources (solar) with excellent flow control abilities of magnetohydrodynamic pumps. Other excellent investigations of this technology include Satyamurthy et al. [31] (for liquid metal solar MHD pumps, based on an extension of the Faraday law of induction to liquid metals), Kaushik et al. [32] (on solar-powered liquid metal LMMHD power generation systems with smaller cost per unit of installed power), Romero and González-Aguilar [33] (on MHD concentrating solar thermal (CST) central tower systems).

Although purely nanofluid media have been exploited to great effect in modern solar and solar MHD pumps, it is also possible to enhance heat transfer characteristics via the incision of a porous material in the pumping duct (channel). Solar porous absorbers have demonstrated exceptional benefits in regulating flows in such pumps and concurrently elevating thermal transfer efficiencies. Wang et al. [34] showed the optimal performance was possible in solar collectors containing a variable porosity medium. Other examples of porous media solar pump systems include the solar pond studied by Shi et al. [35] (where hydrodynamic stability is controlled more effectively with permeability) and Ren et al. [36] who observed that porous media solar pumps and receivers with larger thickness produce better retention of solar thermal energy. Vasiliev et al. [37] have explored the advantages of porous media in solar-gas solid sorption heat pumps. Al-Nimr et al. [38] have analyzed the benefits of porous media matrices in tubeless solar collectors and pumps.

A critical aspect of accurately simulating and designing solar pumps is the proper simulation of thermal radiative heat transfer. Radiation is the dominant mode of heat transfer in sunlight and is the most complex mode of thermal transport. It involves many complex features including spectral effects, optical thickness, reflection, absorption, transmission etc. To simulate radiative heat transfer problems, very sophisticated numerical algorithms must be employed to cater for a 
multitude of thermo-physical phenomena which is both time-consuming and expensive. Many approaches have been developed to overcome this challenge and popular models emerging in engineering sciences include the Milne-Eddington approximation, Chandrasekhar discrete ordinates method, P1 differential approximation, Schuster-Schwartzchild two-flux model and the Rosseland diffusion model. These methods convert the integro-differential radiative equation into either partial differential equations or algebraic flux equations which are much easier to implement. Many simulations have been presented using these radiative models and are lucidly summarized in Tien and Vafai [39]. Radiative (optical) properties of nanofluids are also crucial in their efficient implementation in solar power technology. Said et al. [40] described the application of a direct absorbing nanofluid (suspension formed by mixing nanoparticles and a liquid) for harvesting solar thermal energy, providing extensive details of radiative properties of nanofluids (optical thickness, scattering coefficients etc). Du and Tang [41] elaborated on the transmission and scattering characteristics of nanofluids with agglomeration effects. Bég et al. [42] utilized both network electro-thermal and finite difference algorithms to simulate thermal convection and radiation heat transfer in an annular porous medium solar energy absorber with a Trauggot's P1radiative differential approximation.

Recent trends in engineering design have strongly gravitated towards bio-inspired designs. Biological systems have perfected many intricate mechanisms which can be applied to upgrade conventional engineering systems to a new level of performance and endurance. One of these mechanisms is known as peristalsis and features in an impressive spectrum of natural phenomena. Peristalsis embodies continuous contraction and expansion of a flexible hollow, tubular structure containing fluid. Peristaltic pumping uses this mechanism where direct contact of any inside moving parts with the fluid is undesirable or inefficient. It is ideal for transferring fluids from lower pressure to higher pressure regions. This mechanism is observed in reptilian breathing [43], robotic endoscopy [44], intestinal physiology [47], human speech (laryngeal phonation) [45], and multidrug efflux pumps to export toxic substrates through their cell membranes [46]. Many excellent mathematical models for peristaltic pumping dynamics have been developed. Pal and Brasseur [47] who showed that in esophageal peristalsis, local pressure and shear stress in the contraction zone are strongly diminished via local longitudinal shortening (LLS)and that a peristaltic wave of local longitudinal muscle contraction coordinated with the circular muscle contraction wave has served to concentrate circular muscle fibers and reduces the magnitude of 
contractile force required to transport a parcel of trapped fluid (bolus), collectively enhancing circular muscular efficiency. Tsui et al. [48] conducted numerical computations on peristaltic pumping in a finite length distensible channel in which the unstructured computational grid moved according to the oscillation of the wall. Dobrolyubov and Douchy [49] developed a theory of peristaltic waves based on the travelling deformation waves and wave mass transfer theory. Kumar et al. [50] presented perturbation solutions for peristaltic transport due to a sinusoidal wave travelling on the boundary of a permeable tube filled with an incompressible fluid. Moradi et al. [51] described a spectrally accurate algorithm for peristaltic flows in annular geometries and deployed a numerical discretization method based on Fourier and Chebyshev expansions in the streamwise and radial directions, showing that modifications in the mean axial pressure gradient vary proportionally to the second power of the wave amplitude for waves with small enough amplitudes. Further Newtonian viscous peristaltic pumping modelling studies include Reddy et al. [52] and Mandviwalla and Archer [53]. Ramesh [54] considered magnetohydrodynamic thermosolutal peristaltic pumping of a Stokesian couple stress non-Newtonian fluid in a two-dimensional inclined channel containing a permeable medium. Tripathi and Bég [55] presented closed-form solutions for time-dependent peristaltic magnetohydrodynamic heat transfer through a finite length channel. Nanofluid peristaltic pumping has also been addressed in recent years. Bég and Tripathi [56] presented the first analytical study of thermo-solutal nanofluid peristaltic dynamics in a channel, explicitly considering thermal and species Grashof number buoyancy effects. Further investigations have considered non-Newtonian effects and titanium nano-particles [57], shape geometric effects [58], combined electrical and magnetic field effects [59].

As noted earlier in solar magnetohydrodynamic pumps, radiative heat transfer is a key consideration. The purpose of the present work is to further modify this concept to include biomimetic channel wall features, specifically deformability of the walls. Peristaltic magnetohydrodynamic nanofluid solar pumps must, therefore, feature wall distensibility, nanofluid behavior, magnetohydrodynamics and radiative heat transfer characteristics. The current study uses a Rosseland optically-thick radiative flux model to simulate uni-directional thermal radiation effects. This approach has been utilized in many analyses of peristaltic pumping including Bhatti et al. [60] for two-phase viscoelastic working fluids and Hayat et al. [61] for magnetic nanofluids. A variable-viscosity model is employed for the nanofluid. Darcy's law is employed to simulate porous medium drag effects. Heat source/sink and buoyancy effects are 
included. The conservation equations for mass, momentum, energy and nano-particle volume fraction (concentration) are transformed from a stationary to a moving coordinate system with lubrication theory. The resulting non-dimensional two-point boundary value problem is solved with both a perturbation method and an efficient numerical quadrature technique (Maple 17). The influence of relevant physical parameters on axial velocity, temperature, nanoparticle volume fraction and stream function distributions are depicted and discussed briefly. It is envisaged that the current theoretical and computational model will motivate solar engineers to conduct laboratory investigations of solar MHD nanofluid peristaltic pumps and accelerate the implementation of this promising technology in the near future.

\section{MATHEMATICAL FORMULATION}

Let us consider two-dimensional flow of magnetite nanofluids driven by peristaltic pumping through an asymmetric channel containing a homogenous, isotropic porous wafer material, in the presence of an externally applied transverse magnetic field. The channel walls are propagating with constant wave velocity $(c)$ as sinusoidal wave trains. Let $Y=H_{1}$, and $Y=H_{2}$ be respectively the left and right wall boundaries of the asymmetric channel of width $\left(d_{1}+d_{2}\right)$. The temperature ( $T$ ) and nanoparticle volume fraction $(C)$ are prescribed values of $T_{0}, C_{0}$ and $T_{1}, C_{1}$ at $Y=H_{1}$

and $Y=H_{2}$ respectively. The geometry of the biomimetic solar magnetohydrodynamic nanofluid pump is depicted in Fig.1 and mathematically expressed as:

$$
\begin{aligned}
& H_{1}(X, t)=d_{1}+a_{1} \cos \left(\frac{2 \pi}{\lambda}(X-c t)\right), \\
& H_{2}(X, t)=-d_{2}-a_{2} \cos \left(\frac{2 \pi}{\lambda}(X-c t)+\phi\right) .
\end{aligned}
$$

in which $a_{1}, a_{2}, \lambda, t$ and $\phi$ are the amplitudes of the left and right waves, wavelength, dimensional time and phase difference, further $a_{1}, a_{2}, d_{1}$ and $d_{2}$ satisfy the inlet of the condition:

$$
a_{1}^{2}+a_{2}^{2}+2 a_{1}^{2} a_{2}^{2} \cos \phi \leq\left(d_{1}+d_{2}\right)^{2} .
$$

Buongiorno's [13] model is deployed for the nanofluid. This emphasizes thermophoretic forces and Brownian motion dynamics as the key contributors to thermal conductivity enhancement. This model which has the advantage of being very easily incorporated into the framework of viscous 
fluid dynamics. It also allows the inclusion of a species diffusion (concentration) boundary layer equation which is not possible with other models such as the Tiwari-Das model [14]. It is a twocomponent laminar four-equation non-homogeneous equilibrium model for mass, momentum, and heat transport in nanofluids highlights the dominance of Brownian diffusion and thermophoresis over other nanoscale mechanisms e.g. diffusion-phoresis, Magnus effect, fluid drainage, microconvection and ballistic collisions. The model further assumes that energy transfer via nanoparticle dispersion is negligible and that Dufour (diffuso-thermal) effects may be negated.

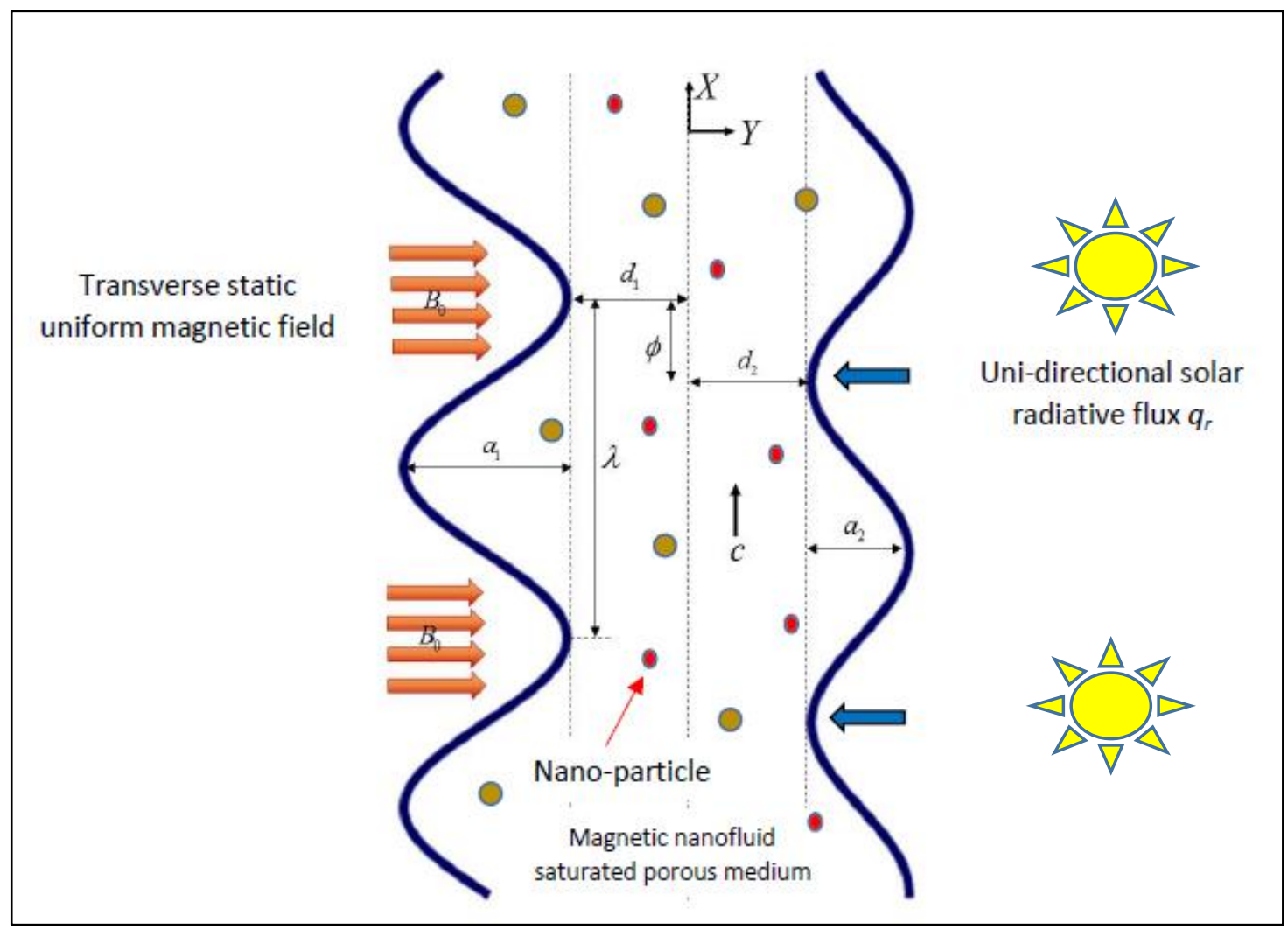

Fig.1. Schematic diagram of the solar magnetic nanofluid peristaltic pump (asymmetric channel).

Implementing the Buongiorno formulation [13] the resulting governing unsteady equations for conservation of mass, momenta (incorporating external magnetic field and Darcy porous matrix resistance), energy and nanoparticle volume fraction (nano-species concentration) in an $(X, Y)$ coordinate system emerge as [62]:

$\frac{\partial U}{\partial X}+\frac{\partial V}{\partial Y}=0$, 


$$
\begin{aligned}
& \rho_{f}\left(\frac{\partial U}{\partial t}+U \frac{\partial U}{\partial X}+V \frac{\partial U}{\partial Y}\right)=-\frac{\partial P}{\partial X}+2 \frac{\partial}{\partial X}\left[\mu(T) \frac{\partial U}{\partial X}\right]+\frac{\partial}{\partial Y}\left[\mu(T)\left(\frac{\partial V}{\partial X}+\frac{\partial U}{\partial Y}\right)\right]-\frac{\mu(T)}{k_{0}} U \\
&-\sigma^{\prime} B_{0}^{2} U+\left(1-C_{0}\right) \rho_{0} g \beta_{t}\left(T-T_{0}\right)-\left(\rho_{p}-\rho_{0}\right) g\left(C-C_{0}\right), \\
& \rho_{f}\left(\frac{\partial V}{\partial t}+U \frac{\partial V}{\partial X}+V \frac{\partial V}{\partial Y}\right)=-\frac{\partial P}{\partial Y}+2 \frac{\partial}{\partial Y}\left[\mu(T) \frac{\partial V}{\partial Y}\right]+\frac{\partial}{\partial X}\left[\mu(T)\left(\frac{\partial V}{\partial X}+\frac{\partial U}{\partial Y}\right)\right]-\frac{\mu(T)}{k_{0}} U, \\
&\left(\rho C^{\prime}\right)_{f}\left(\frac{\partial T}{\partial t}+U \frac{\partial T}{\partial X}+V \frac{\partial T}{\partial Y}\right)=k_{e f}\left[\frac{\partial^{2} T}{\partial X^{2}}+\frac{\partial^{2} T}{\partial Y^{2}}\right]+\left(\rho C^{\prime}\right)_{p}\left[D_{B}\left(\frac{\partial C}{\partial X} \frac{\partial T}{\partial X}+\frac{\partial C}{\partial Y} \frac{\partial T}{\partial Y}\right)\right]+ \\
&\left(\frac{\partial C}{\partial t}+U \frac{\partial C}{\partial X}+V \frac{\partial C}{\partial Y}\right)=D_{B}\left[\frac{D^{2} C}{\partial X^{2}}+\frac{\partial^{2} C}{\partial Y^{2}}\right]+\frac{D_{T}}{T_{0}}\left[\frac{\partial^{2} T}{\partial X^{2}}+\frac{\partial^{2} T}{\partial Y^{2}}\right]
\end{aligned}
$$

where, $U, V$ are the velocity components in $X, Y$ directions, $\rho_{f}$ is fluid density, $P$ is pressure, $\mu(T)$ is temperature dependent viscosity, $k_{0}$ is the permeability parameter, $\sigma^{\prime}$ is electrical conductivity, $B_{o}$ is transverse magnetic field, $g$ isacceleration due to gravity, $\beta_{t}$ is volumetric thermal expansion coefficient of the fluid, $\beta_{c}$ is volumetric solutal expansion coefficient of the fluid, $\rho_{0}$ is the nanofluid density at the reference temperature $\left(T_{0}\right), \rho_{p}$ is nanoparticle mass density, $\left(\rho C^{\prime}\right)_{f}$ is heat capacity of fluid, $k_{e f}$ is thermal conductivity, $\left(\rho C^{\prime}\right)_{p}$ is effective heat capacity of nanoparticle, $D_{B}$ isBrownian diffusion coefficient, $D_{T}$ is thermophoretic diffusion coefficient,,$Q_{0}$ is the constant heat addition/absorption. We further note that in the solar MHD nanofluid pump circuit, the direction of the current density $\mathrm{J}$ determines whether the system behaves as a pump or as a generator. When electric current passes through an electrically-neutral conducting medium in the presence of a magnetic field, a vector body force per unit volume $F\left(N / \mathrm{m}^{3}\right)$ affects the medium. $\mathrm{F}$ is referred to as Lorentz force and is given by $-\sigma B_{o}{ }^{2} u$ i.e. the fifth term on the right-hand side of the X-momentum equation (4). We consider the case where the current density accelerates the 
flow i.e. works as a pump (the opposite case of a generator is produced when the current density is reversed and as a result so is the Lorentz body force).We neglect magnetic field leakage and assume that the magnetic field is uniform during operation. Since the magnetic Reynolds number of the flow is taken to be very small, the induced magnetic field is negligible and it is further assumed that no external electric field is applied. Therefore, the electric field due to polarization of charges is negligible so that no energy is added or extracted from the fluid by electrical means. Ohmic (Joule) heating is neglected as are Soret and Dufour cross-diffusion effects. The radiative heat flux $\left(q_{r}\right)$ is given by using Rosseland's approximation. This approximation is valid for optically-thick fluids which can absorb or emit radiation at their boundaries [1].

$$
q_{r}=-\frac{4 \sigma^{*}}{3 k^{*}} \frac{\partial T^{4}}{\partial Y} .
$$

Here $\sigma^{*}$ and $k^{*}$ are the Stefan-Boltzmann constant and the mean absorption coefficient, respectively. We assume that the temperature difference within the flow is adequately small. The term $T^{4}$ is expanded as a Taylor series about a free stream temperature $T_{0}$ and ignoring higher order terms in the first order in $\left(T-T_{0}\right)$, we get:

$$
q_{r}=-\frac{16 \sigma * T_{0}^{3}}{3 k *} \frac{\partial T}{\partial Y} .
$$

The transformations from the fixed frame of reference $(X, Y)$ to the wave frame of reference $(\bar{x}$, $\bar{y})$ are given by:

$$
\bar{x}=X-c \bar{t}, \bar{y}=Y, \bar{u}=U-c, \bar{v}=V \text { and } p(\bar{x})=P(X, \bar{t}) .
$$

After utilizing the transformations (10), equations (1) - (7) retract to the following form:

$$
\begin{aligned}
\frac{\partial \bar{u}}{\partial \bar{x}}+\frac{\partial \bar{v}}{\partial \bar{y}}=0, & \begin{aligned}
\rho_{f}\left[(\bar{u}+c) \frac{\partial \bar{u}}{\partial \bar{x}}+\bar{v} \frac{\partial \bar{u}}{\partial \bar{y}}\right]= & -\frac{\partial \bar{p}}{\partial \bar{x}}+2 \frac{\partial}{\partial \bar{x}}\left[\mu(T) \frac{\partial \bar{u}}{\partial \bar{x}}\right]+\frac{\partial}{\partial \bar{y}}\left[\mu(T)\left(\frac{\partial \bar{v}}{\partial \bar{x}}+\frac{\partial \bar{u}}{\partial \bar{y}}\right)\right]-\frac{\mu(T)}{k_{0}} \bar{u} \\
& -\sigma^{\prime} B_{0}^{2} \bar{u}+\left(1-C_{0}\right) \rho_{0} g \beta_{t}\left(T-T_{0}\right)-\left(\rho_{p}-\rho_{0}\right) g\left(C-C_{0}\right),
\end{aligned}
\end{aligned}
$$




$$
\begin{aligned}
& \rho_{f}\left[(\bar{u}+c) \frac{\partial \bar{v}}{\partial \bar{x}}+\bar{v} \frac{\partial \bar{v}}{\partial \bar{y}}\right]=-\frac{\partial \bar{p}}{\partial \bar{y}}+2 \frac{\partial}{\partial \bar{y}}\left[\mu(T) \frac{\partial \bar{v}}{\partial \bar{y}}\right]+\frac{\partial}{\partial \bar{x}}\left[\mu(T)\left(\frac{\partial \bar{v}}{\partial \bar{x}}+\frac{\partial \bar{u}}{\partial \bar{y}}\right)\right]-\frac{\mu(T)}{k_{0}} \bar{v}, \\
& \left(\rho C^{\prime}\right)_{f}\left[(\bar{u}+c) \frac{\partial T}{\partial \bar{x}}+v \frac{\partial T}{\partial \bar{y}}\right]=k_{e f}\left[\frac{\partial^{2} T}{\partial \bar{x}^{2}}+\frac{\partial^{2} T}{\partial \bar{y}^{2}}\right]+\left(\rho C^{\prime}\right)_{p}\left[D_{B}\left(\frac{\partial C}{\partial \bar{x}} \frac{\partial T}{\partial \bar{x}}+\frac{\partial C}{\partial \bar{y}} \frac{\partial T}{\partial \bar{y}}\right)\right] \\
& +\frac{D_{T}}{T_{0}}\left[\left(\frac{\partial T}{\partial \bar{x}}\right)^{2}+\left(\frac{\partial T}{\partial \bar{y}}\right)^{2}\right]+Q_{0}-\frac{\partial q_{r}}{\partial \bar{y}} \\
& {\left[(\bar{u}+c) \frac{\partial C}{\partial \bar{x}}+v \frac{\partial C}{\partial \bar{y}}\right]=D_{B}\left[\frac{\partial^{2} C}{\partial \bar{x}^{2}}+\frac{\partial^{2} C}{\partial \bar{y}^{2}}\right]+\frac{D_{T}}{T_{0}}\left[\frac{\partial^{2} T}{\partial \bar{x}^{2}}+\frac{\partial^{2} T}{\partial \bar{y}^{2}}\right]}
\end{aligned}
$$

We now introduce the following dimensionless variables:

$$
\begin{aligned}
& x=\frac{\bar{x}}{\lambda}, y=\frac{\bar{y}}{d_{1}}, u=\frac{\bar{u}}{c}, v=\frac{\lambda \bar{v}}{d_{1} c}, p=\frac{d_{1}^{2} p}{\mu_{0} c \lambda}, t=\frac{c \bar{t}}{\lambda}, h_{1}(x)=\frac{H_{1}(\bar{x})}{d_{1}}, h_{2}(x)=\frac{H_{2}(\bar{x})}{d_{1}}, a=\frac{a_{1}}{d_{1}}, b=\frac{a_{2}}{d_{1}}, \\
& d=\frac{d_{2}}{d_{1}}, M^{2}=\frac{\sigma^{\prime} B_{0}^{2} d_{1}^{2}}{\mu_{0}}, \theta=\frac{T-T_{0}}{T_{1}-T_{0}}, \sigma=\frac{C-C_{0}}{C_{1}-C_{0}}, \mu(\theta)=\frac{\mu(T)}{\mu_{0}}, R=\frac{\rho_{f} c d_{1}}{\mu_{0}}, K=\frac{k_{0}}{d_{1}^{2}}, \\
& G r=\frac{\left(1-C_{0}\right) \rho_{0}^{2} g \beta_{t} d_{1}^{3}\left(T_{1}-T_{0}\right)}{\mu_{0}^{2}}, B r=-\frac{\rho_{0}\left(\rho_{p}-\rho_{0}\right) g d_{1}^{3}\left(C_{1}-C_{0}\right)}{\mu_{0}^{2}}, \operatorname{Pr}=\frac{C_{f}^{\prime} \mu_{0}}{k_{e f}}, R n=\frac{16 \sigma^{*} T_{0}^{3}}{3 k^{*} \mu_{0} C_{f}{ }^{\prime}}, \\
& \beta=\frac{d_{1}^{2} Q_{0}}{\left(T_{1}-T_{0}\right) \mu_{0} C_{f}{ }^{\prime}}, N t=\frac{\left(T_{1}-T_{0}\right) D_{T}\left(\rho C^{\prime}\right)_{p}}{k_{e f} T_{0}}, N b=\frac{\left(C_{1}-C_{0}\right)\left(\rho C^{\prime}\right)_{p} D_{B}}{k_{e f}} .
\end{aligned}
$$

Here $R, R n, d, a$ and $b, M, K, G r, B r, \operatorname{Pr}, \beta, N b, N t, \theta$ and $\sigma$ are the Reynolds number, radiation parameter, width of the channel, amplitudes of left and right walls, Hartmann number, permeability parameter, thermal Grashof number, local nanoparticle Grashof number, Prandtl number, heat source/sink parameter, Brownian motion parameter, thermophoresis parameter, non-dimensional temperature and nano-particle volume fraction, respectively.

Applying the long wavelength and low Reynolds number approximations and introducing the stream function $u=\frac{\partial \psi}{\partial y}$, eqns. (12-15) reduce to:

$$
\begin{aligned}
& 0=-\frac{\partial p}{\partial x}+\frac{\partial}{\partial y}\left[\mu(\theta) \frac{\partial^{2} \psi}{\partial y^{2}}\right]-\left(\frac{1}{K}+M^{2}\right)\left(\frac{\partial \psi}{\partial y}+1\right)+G r \theta+B r \sigma \\
& 0=-\frac{\partial p}{\partial y}
\end{aligned}
$$




$$
\begin{aligned}
& 0=\left(\frac{1}{\operatorname{Pr}}+R n\right) \frac{\partial^{2} \theta}{\partial y^{2}}+N b\left(\frac{\partial \sigma}{\partial y} \frac{\partial \theta}{\partial y}\right)+N t\left(\frac{\partial \theta}{\partial y}\right)^{2}+\beta, \\
& 0=\frac{\partial^{2} \sigma}{\partial y^{2}}+\frac{N t}{N b} \frac{\partial^{2} \theta}{\partial y^{2}} .
\end{aligned}
$$

Eqn.(18) implies that $\mathrm{p}$ is not function of $y$. Differentiating Eqn. (17) with respect to $y$, yields:

$$
0=\frac{\partial^{2}}{\partial y^{2}}\left[\mu(\theta) \frac{\partial^{2} \psi}{\partial y^{2}}\right]-N^{2} \frac{\partial^{2} \psi}{\partial y^{2}}+G r \theta+B r \sigma,
$$

Where $N^{2}=\frac{1}{K}+M^{2}$.

The volumetric flow rate in the wave frame is given by:

$$
q=\int_{h_{1}}^{h_{2}} u d y=\int_{h_{1}}^{h_{2}}(U-1) d y
$$

On integration, eqn. (22) yields:

$$
q=Q+h_{1}-h_{2}
$$

Averaging the volumetric flow rate along one time-period, we get:

$$
\Theta=\int_{0}^{1} Q d t=\int_{0}^{1}\left(q+h_{2}-h_{1}\right) d t
$$

which yields:

$$
\Theta=q+1+d .
$$

The appropriate boundary conditions may be presented in the following form:

$$
\begin{aligned}
& \psi=\frac{q}{2}, \frac{\partial \psi}{\partial y}=-1, \theta=0 \text { and } \sigma=0 \text { at } y=h_{1}(x)=1+a \cos 2 \pi x, \\
& \psi=-\frac{q}{2}, \frac{\partial \psi}{\partial y}=-1, \theta=1 \text { and } \sigma=1 \text { at } y=h_{2}(x)=-d-b \cos (2 \pi x+\phi),
\end{aligned}
$$

which satisfy the condition: $a^{2}+b^{2}+2 a b \cos (\phi) \leq(1+d)^{2}$.

Solving the Eqns. (19-20) subject to boundary condition (26), the temperature and nanoparticle fraction field are obtained as:

$$
\begin{aligned}
& \theta(y)=A_{3} e^{-A_{1} y}-\frac{A_{2} y}{A_{1}}-A_{4}, \\
& \sigma(y)=A_{5} y-A_{6} e^{-A_{1} y}-A_{7} .
\end{aligned}
$$




\section{PERTURBATION SOLUTION}

Although in most previous studies of fluid mechanical problems for the sake of simplicity of the analysis, fluid viscosity has been taken to be constant, in many real MHD pump flows the viscosity is a function of temperature. Keeping this in mind, in the present study temperature-dependent fluid viscosity has been included and simulated as a linear function of temperature. Thus, for the analysis, we adopt Reynolds' model of viscosity as:

$$
\mu(\theta)=1-\alpha \theta \quad \text { for } \quad \alpha<<1 .
$$

Applying Eqn. (29) to Eqn. (21), we obtain:

$$
0=(1-\alpha \theta) \frac{\partial^{4} \psi}{\partial y^{4}}-2 \alpha \frac{\partial \theta}{\partial y} \frac{\partial^{3} \psi}{\partial y^{3}}-\left(\alpha \frac{\partial^{2} \theta}{\partial y^{2}}+N^{2}\right) \frac{\partial^{2} \psi}{\partial y^{2}}+G r \frac{\partial \theta}{\partial y}+B r \frac{\partial \sigma}{\partial y}
$$

The case for a nanofluid with constant viscosity can be retrieved as a special case of the present investigation when we assume $\alpha=0$.Eqn.(30) is a highly nonlinear partial differential equation and it is not possible to derive exact solutions. However we may deploy a perturbation method in terms of $\alpha$ (viscosity parameter), by expanding $\psi$ and $F$ in the following forms:

$$
\begin{aligned}
& \psi=\psi_{0}+\alpha \psi_{1}+o\left(\psi_{2}\right), \\
& F=F_{0}+\alpha F_{1}+o\left(F_{2}\right) .
\end{aligned}
$$

Inserting the above expressions into Eqn. (30) and boundary conditions Eqn. (26), the following systems emerge:

\subsection{For the system of order $\left(\alpha^{0}\right)$}

$$
\begin{aligned}
& \frac{\partial^{4} \psi_{0}}{\partial y^{4}}-N^{2} \frac{\partial^{2} \psi_{0}}{\partial y^{2}}+G r \frac{\partial \theta}{\partial y}+B r \frac{\partial \sigma}{\partial y}=0, \\
& \psi_{0}=\frac{q_{0}}{2}, \frac{\partial \psi_{0}}{\partial y}=-1 \text { at } y=h_{1}, \\
& \psi_{0}=-\frac{q_{0}}{2}, \frac{\partial \psi_{0}}{\partial y}=-1 \text { at } y=h_{2} .
\end{aligned}
$$

\subsection{For the system of $\operatorname{order}\left(\alpha^{1}\right)$}

$$
\frac{\partial^{4} \psi_{1}}{\partial y^{4}}-\theta \frac{\partial^{4} \psi_{0}}{\partial y^{4}}-2 \frac{\partial \theta}{\partial y} \frac{\partial^{3} \psi_{0}}{\partial y^{3}}-\frac{\partial^{2} \theta}{\partial y^{2}} \frac{\partial^{2} \psi_{0}}{\partial y^{2}}-N^{2} \frac{\partial^{2} \psi_{1}}{\partial y^{2}}=0
$$




$$
\begin{aligned}
& \psi_{1}=\frac{q_{1}}{2}, \frac{\partial \psi_{1}}{\partial y}=0 \text { at } y=h_{1}, \\
& \psi_{1}=-\frac{q_{1}}{2}, \frac{\partial \psi_{1}}{\partial y}=0 \text { at } \quad y=h_{2} .
\end{aligned}
$$

\subsection{Solution for system of order $\left(\alpha^{0}\right)$}

Solution of Eqn. (33) subject to boundary conditions Eq.(34a\&b) can be derived as:

$$
\psi_{0}(y)=c_{5}+c_{6} e^{-N y}+L_{10} y^{2}-\frac{c_{8} y}{N^{2}}+L_{11} e^{-A_{1} y}+c_{7} e^{N y} .
$$

\subsection{Solution for system of $\operatorname{order}\left(\alpha^{1}\right)$}

Using Eqn.(37) into Eqn.(35) and invoking the boundary conditions (36 a \& b), we arrive at:

$$
\begin{aligned}
\psi_{1}(y)= & c_{9}+c_{10} e^{-N y}+c_{11} e^{N y}-\frac{c_{12} y}{N^{2}}+L_{1} e^{-A_{1} y}+L_{2}+L_{3} e^{-2 A_{1} y}+L_{4} y e^{-A_{1} y}+L_{5} e^{-N y} \\
& +L_{6} e^{N y}+L_{7} y e^{N y}+L_{8} e^{-\left(A_{1}+N\right) y}+L_{9} e^{-\left(A_{1}-N\right) y}
\end{aligned}
$$

The stream function of the nanofluid is given by the expression:

$$
\begin{aligned}
\psi & \psi_{0}+\alpha \psi_{1}+o\left(\psi_{2}\right) \\
& +\alpha(y)=c_{5}+c_{6} e^{-N y}+L_{10} y^{2}-\frac{c_{8} y}{N^{2}}+L_{11} e^{-A_{1} y}+c_{7} e^{N y} \\
& +\alpha\left(\begin{array}{l}
c_{9}+c_{10} e^{-N y}+c_{11} e^{N y}-\frac{c_{12} y}{N^{2}}+L_{1} e^{-A_{1} y}+L_{2}+L_{3} e^{-2 A_{1} y}+L_{4} y e^{-A_{1} y} \\
+L_{5} e^{-N y}+L_{6} e^{N y}+L_{7} y e^{N y}+L_{8} e^{-\left(A_{1}+N\right) y}+L_{9} e^{-\left(A_{1}-N\right) y}
\end{array}\right) .
\end{aligned}
$$

Axial velocity can also be derived as:

$$
\begin{aligned}
u(y)=2 L_{10} y-\frac{c_{8}}{N^{2}}-A_{1} L_{11} e^{-A_{1} y}-c_{6} N e^{-N y}+c_{7} N e^{N y} \\
+\alpha\left(\begin{array}{l}
\left(L_{7}+L_{7} N y+c_{11} N+L_{6} N\right) e^{N y}-\frac{c_{12}}{N^{2}}+L_{4} \mathrm{e}^{-A_{1} y}-\left(c_{10}+L_{5}\right) N e^{-N y} \\
-L_{8}\left(N+A_{1}\right) e^{-\left(N+A_{1}\right) y}+L_{9}\left(N-A_{1}\right) \mathrm{e}^{\left(N-A_{1}\right) y}-2 L_{3} A_{1} \mathrm{e}^{-2 A_{1} y} \\
-\left(L_{4} y+L_{1}\right) A_{1} \mathrm{e}^{-A_{1} y}
\end{array}\right) .
\end{aligned}
$$

All the coefficients featured in the above closed-form solutions, are presented in the Appendix. 


\section{VALIDATION WITH MAPLE17}

The linear dimensionless two-point moving boundary value problem (BVP) i.e. eqns. (19), (20) and (21) with conditions (26a, 26b) are easily solved using Runge-Kutta-Merson numerical quadrature to yield temperature, nano-particle volume fraction and stream function. The axial velocity is then computed in a sub-iteration loop based on the definition $u=\frac{\partial \psi}{\partial y}$. The computations are executed in MAPLE17 software (RK45 algorithm). This approach has been extensively implemented recently in non-Newtonian nanofluid flows [63].The robustness and stability of this numerical method is therefore well established-it is highly adaptive since it adjusts the quantity and location of grid points during iteration and thereby constrains the local error within acceptable specified bounds. In the current problem, the wall boundary conditions given in Eqns. (26a, b) are easily accommodated. The stepping formulae although designed for nonlinear problems, are even more efficient for any order of linear differential equation and are summarized below [63]:

$$
\begin{aligned}
& k_{0}=f\left(x_{i}, y_{i}\right), \\
& k_{1}=f\left(x_{i}+\frac{1}{4} h, y_{i}+\frac{1}{4} h k_{0}\right), \\
& k_{2}=f\left(x_{i}+\frac{3}{8} h, y_{i}+\left(\frac{3}{32} k_{0}+\frac{9}{32} k_{1}\right) h\right), \\
& k_{3}=f\left(x_{i}+\frac{12}{13} h, y_{i}+\left(\frac{1932}{2197} k_{0}-\frac{7200}{2197} k_{1}+\frac{7296}{2197} k_{2}\right) h\right), \\
& k_{4}=f\left(x_{i}+h, y_{i}+\left(\frac{439}{216} k_{0}-8 k_{1}+\frac{3860}{513} k_{2}-\frac{845}{4104} k_{3}\right) h\right), \\
& k_{i+1}=y_{i}+\left(\frac{25}{216} k_{0}+\frac{1408}{2565} k_{2}+\frac{2197}{4101} k_{3}-\frac{1}{5} k_{4}\right) h, \\
& z_{i+1}=z_{i}+\left(\frac{16}{135} k_{0}+\frac{6656}{12825} k_{2}+\frac{28561}{56430} k_{3}-\frac{9}{50} k_{4}+\frac{2}{55} k_{5}\right) h .
\end{aligned}
$$

Here $y$ denotes fourth-order Runge-Kutta phase and zis the fifth-order Runge-Kutta phase. An estimate of the error is achieved by subtracting the two values obtained. If the error exceeds a 
specified threshold, the results can be re-calculated using a smaller step size. The approach to estimating the new step size is shown below:

$$
h_{\text {new }}=h_{\text {old }}\left(\frac{\varepsilon h_{\text {old }}}{2\left|z_{i+1}-y_{i+1}\right|}\right)^{1 / 4} \text {. }
$$

A comparison of the analytical and MAPLE numerical quadrature solutions (blue dots) is documented in Figs. 2a-ffor pressure rise profile with variation in (a) $\alpha$, (b) $M$, (c) $K$, (d) $R n$, (e) $\beta$ and (f) $\mathrm{Br}$ and also Figs. 3a-f for axial velocity distributions (axial velocity vs. transverse coordinate) for (a) $M$, (b) $R n$, (c) $\operatorname{Pr}$, (d) $K$, (e) $\beta$, (f) $\alpha$ and finally in Figs $4 a-d$ for temperature and nanoparticle volume fraction profiles with variation in (a) $\beta$, (b) $N t$, (c) $\operatorname{Pr}$, (d) $R n$. Only one case is validated in each plot. Excellent correlation is achieved in all cases. Confidence in the present analytical solutions is therefore high. Maple quadrature is of comparable accuracy to many other sophisticated semi-numerical methods including homotopy analysis methods (HAM), Adomian decomposition methods (ADM), spectral collocation Chebyschev polynomial methods and Variational iterative methods (VIMs) which accurately compute series solution, although Maple quadrature is less algebraically rigorous and can be applied directly for all types of differential and integral equations, linear or nonlinear, homogeneous or inhomogeneous, with constant coefficients or with variable coefficients. Another important advantage is that the method is capable of greatly reducing the size of computation work while still maintaining high accuracy of the numerical solution [63].

\section{NUMERICAL EVALUATION OF RESULTS AND DISCUSSION}

The primary aim of this investigation is to analyze the influence of temperature-dependence of the nanoliquid viscosity, thermal radiative flux, wafer permeability and heat source/sink effects on thermal flow characteristics of the solar peristaltic nanofluid magnetohydrodynamic micro-pump. In this regard, herein we elaborate on the impact of various pertinent hydrodynamic, magnetic, thermal and porous medium parameters i.e. Reynolds number viscosity parameter $(\alpha)$, heat source/sink parameter $(\beta)$, Prandtl number $(\operatorname{Pr})$, Hartmann number $(M)$, thermal radiation $(R n)$, Permeability parameter $(K)$ and local nanoparticle Grashof number $(B r)$ at a pre-determined axial location along the pump channel $(x=0.5)$ on the evolution of axial velocity of the fluid $(u)$, 
pressure gradient $(d p / d x)$, temperature distribution $(\theta)$ and nanoparticle volume fraction of the fluid $(\sigma)$. The distributions are depicted in Figs.2-7. Evaluation of the closed form solutions is conducted via the symbolic software Mathematica which is very versatile for peristaltic and hydromagnetic pump simulations.
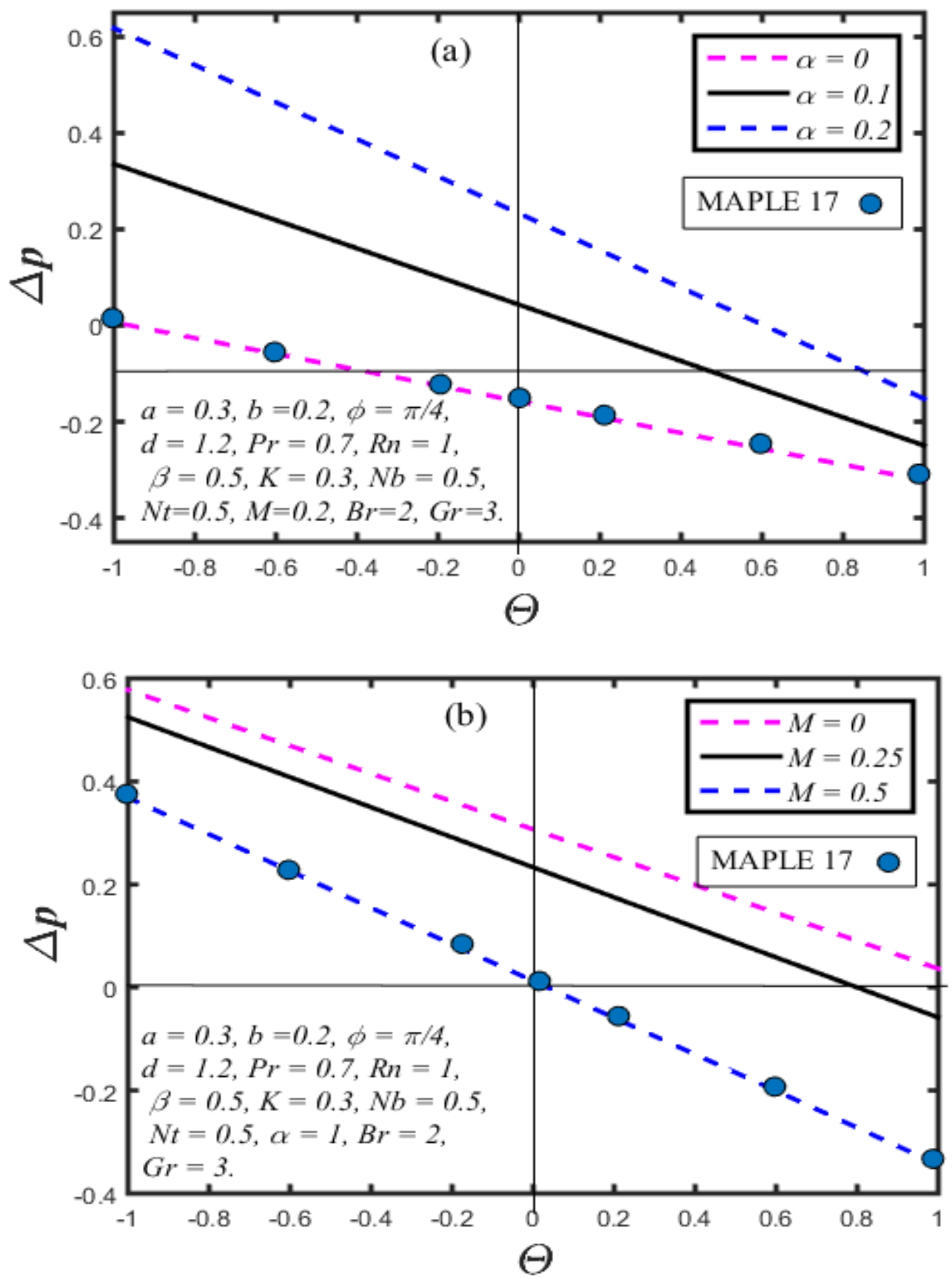

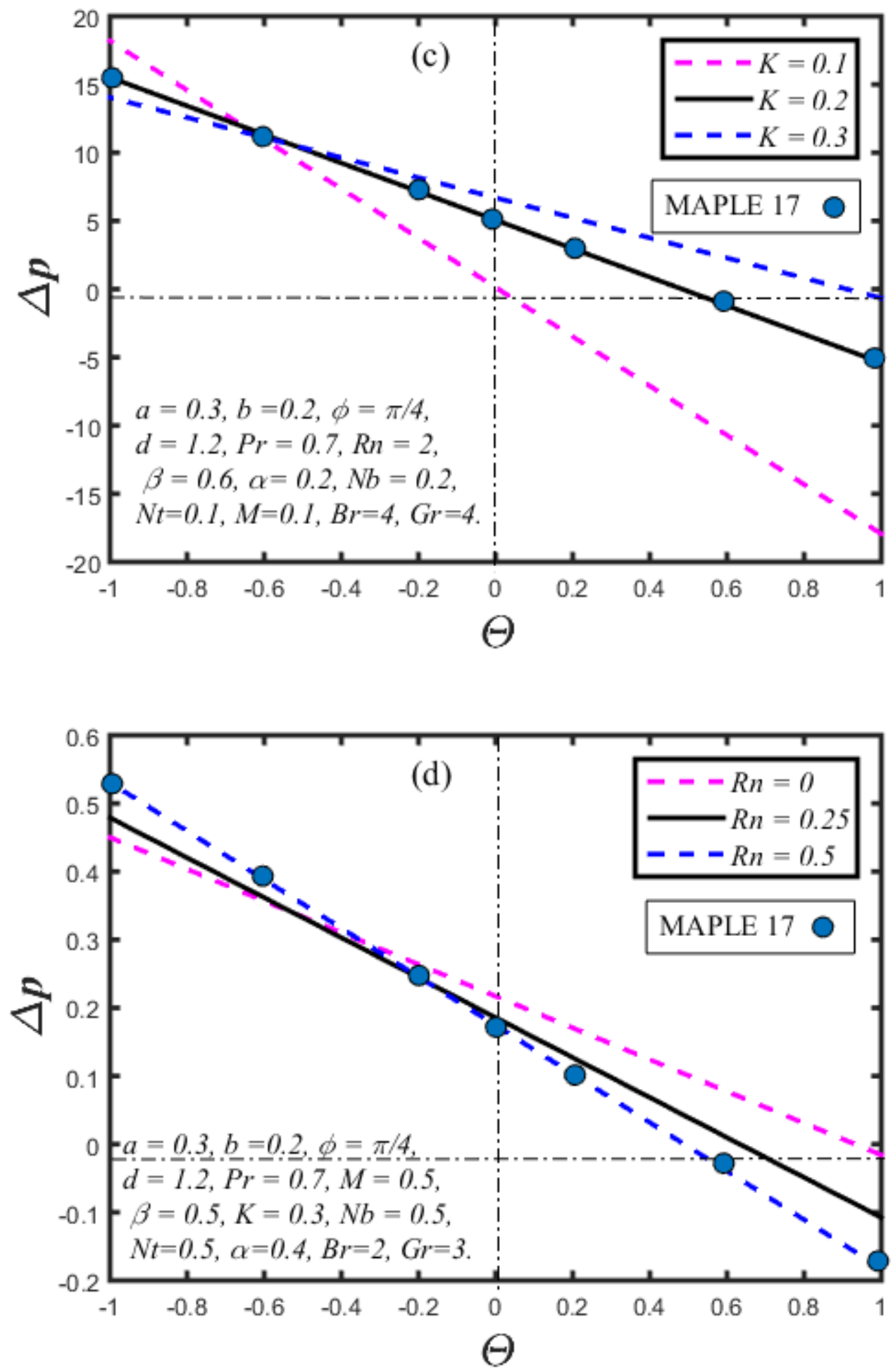

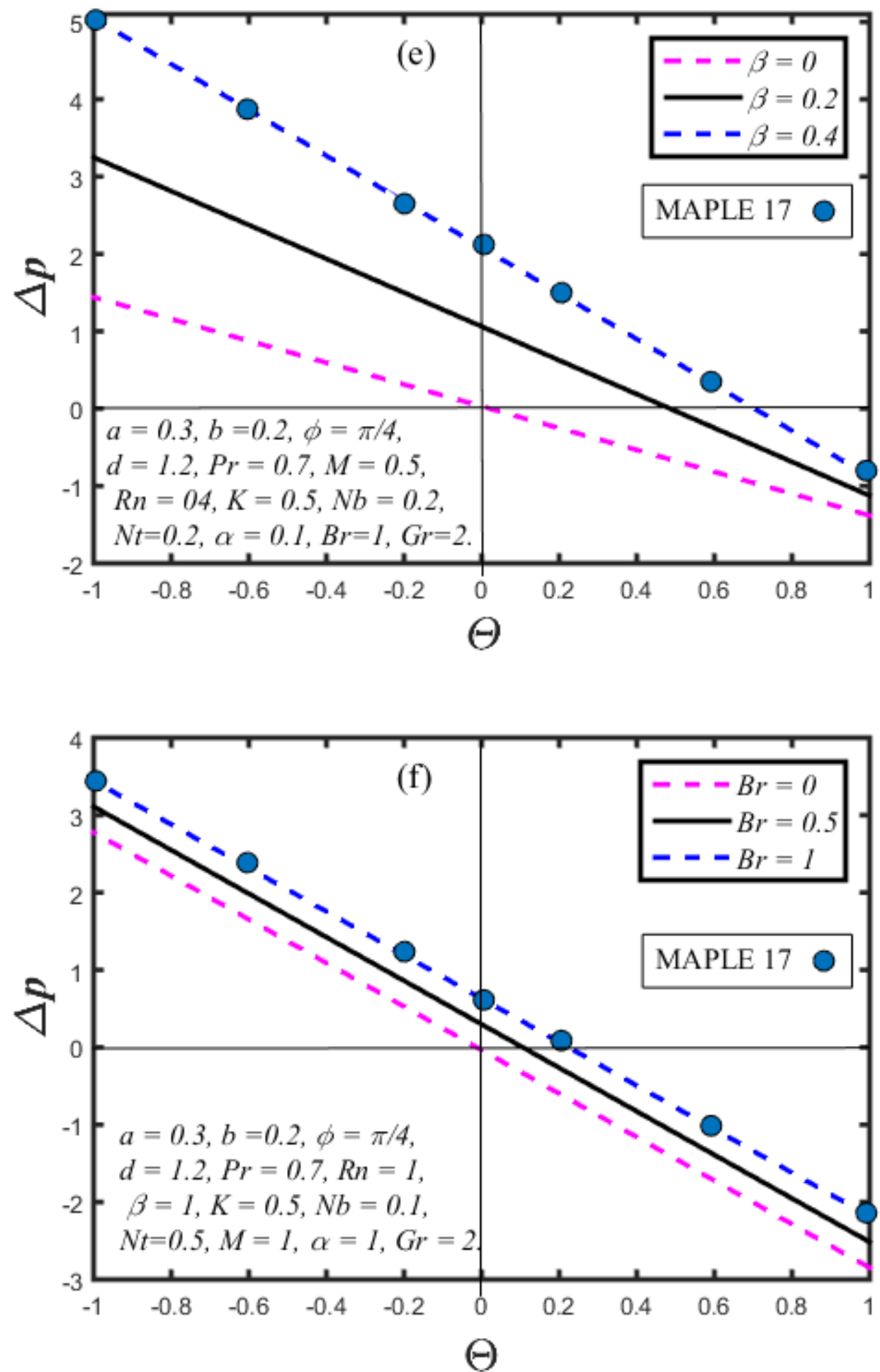

Fig.2. Pressure rise profile with variation in (a) $\alpha$, (b) $M$, (c) $K$, (d) $R n$, (e) $\beta$ and (f) $B r$. 

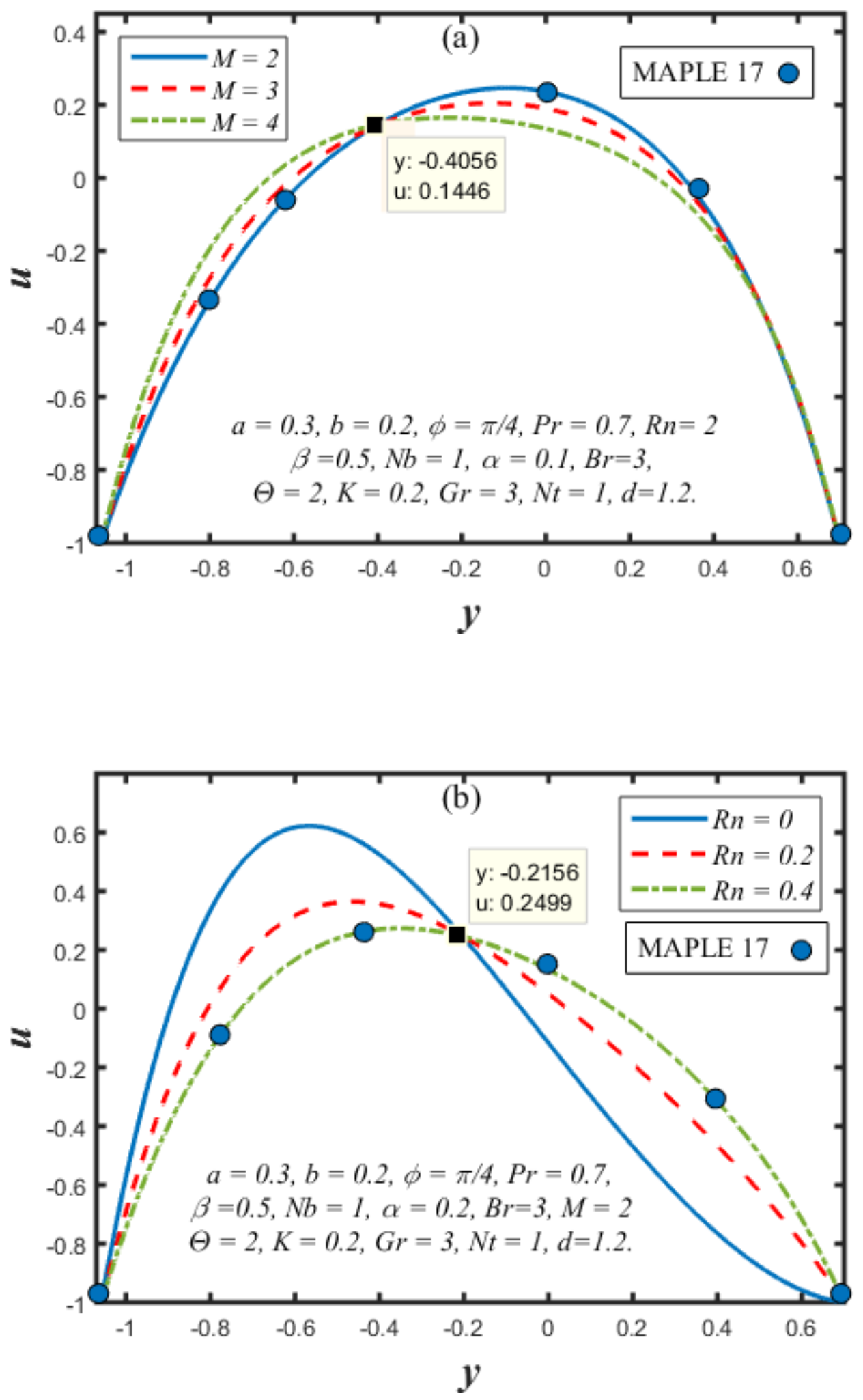

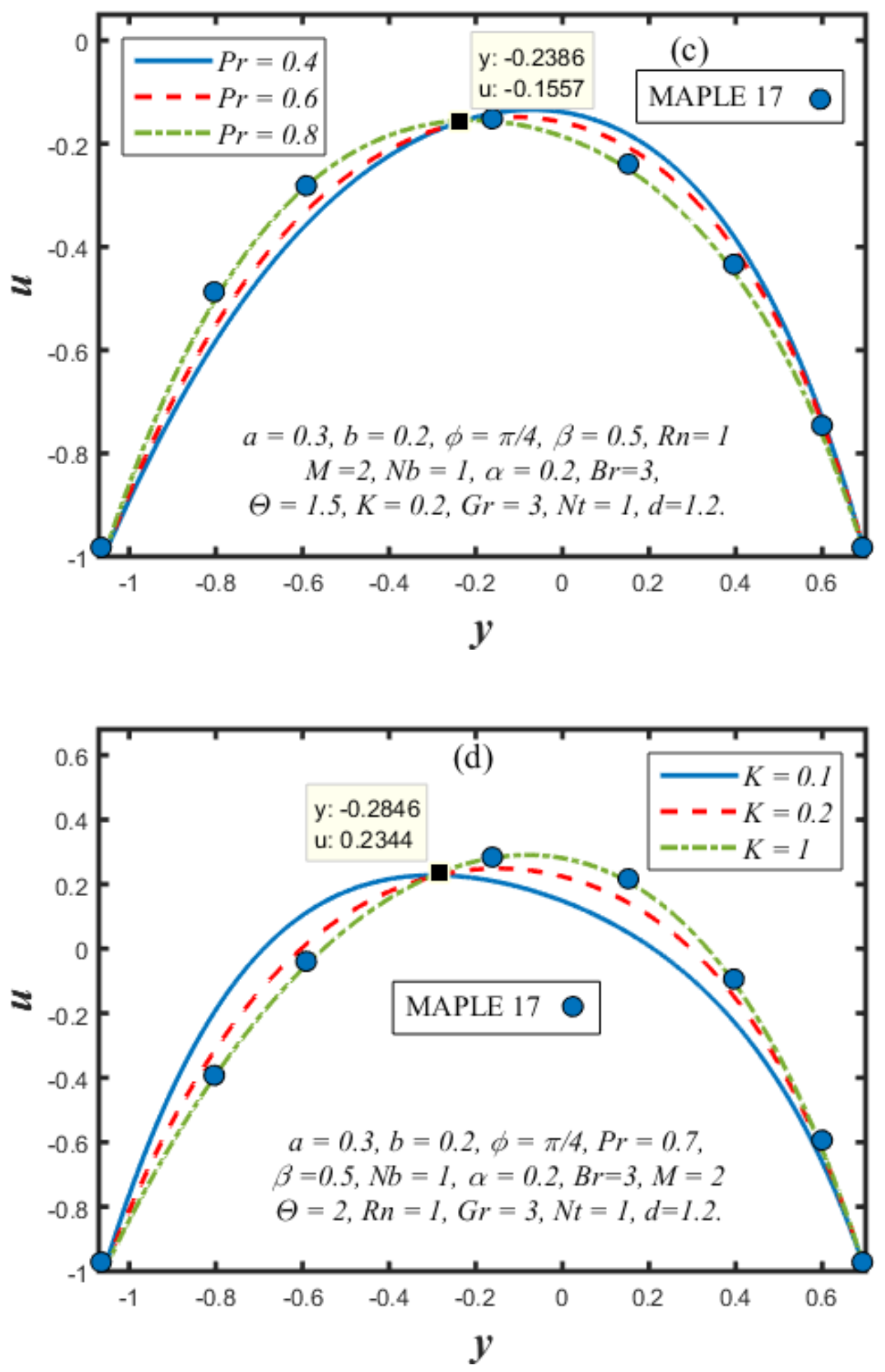

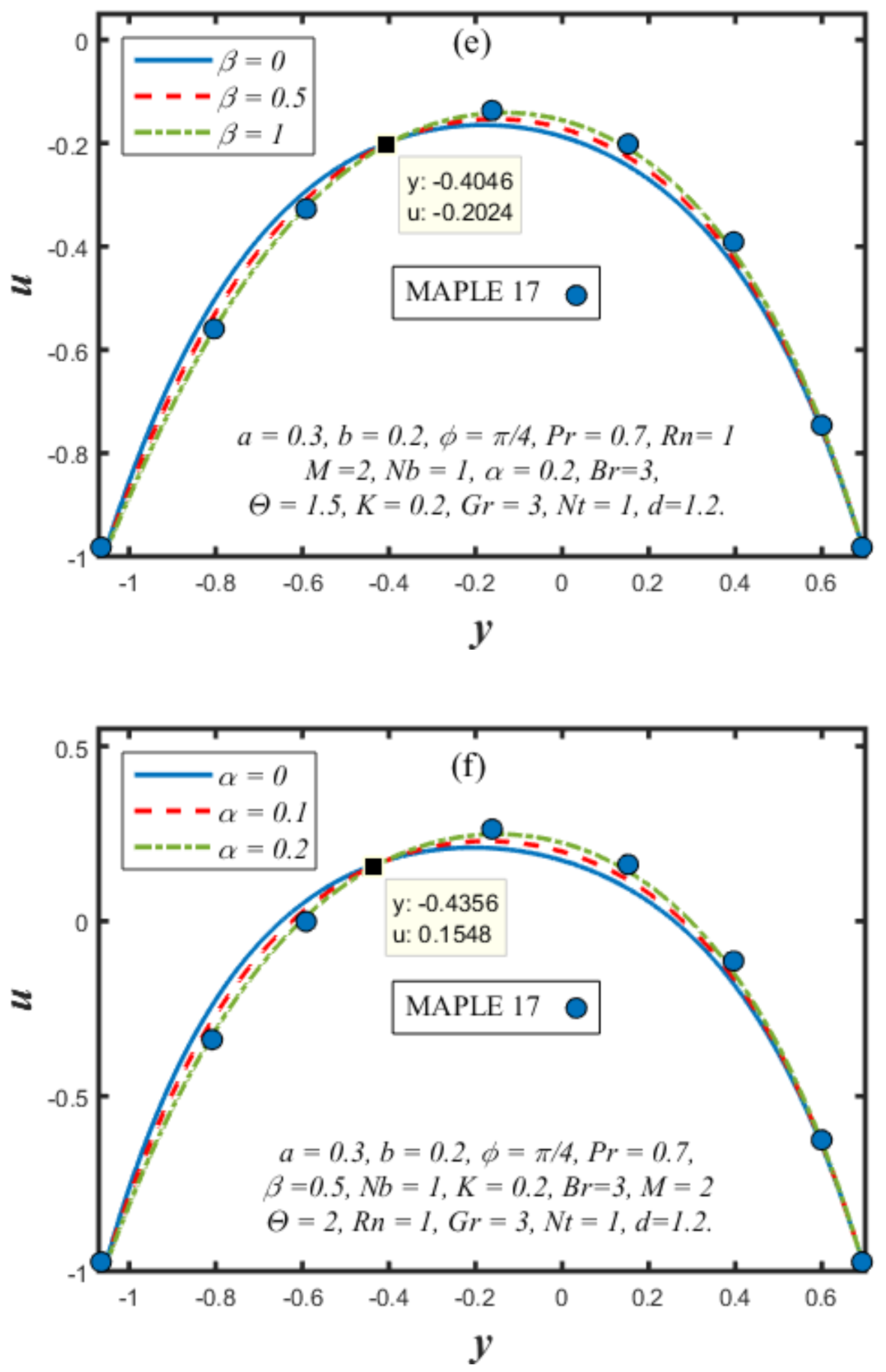

Fig.3. Axial velocity distributions (axial velocity vs. transverse coordinate) for (a) $M$, (b) $R n$, (c) $\operatorname{Pr}$, (d) $K$, (e) $\beta$, (f) $\alpha$. 

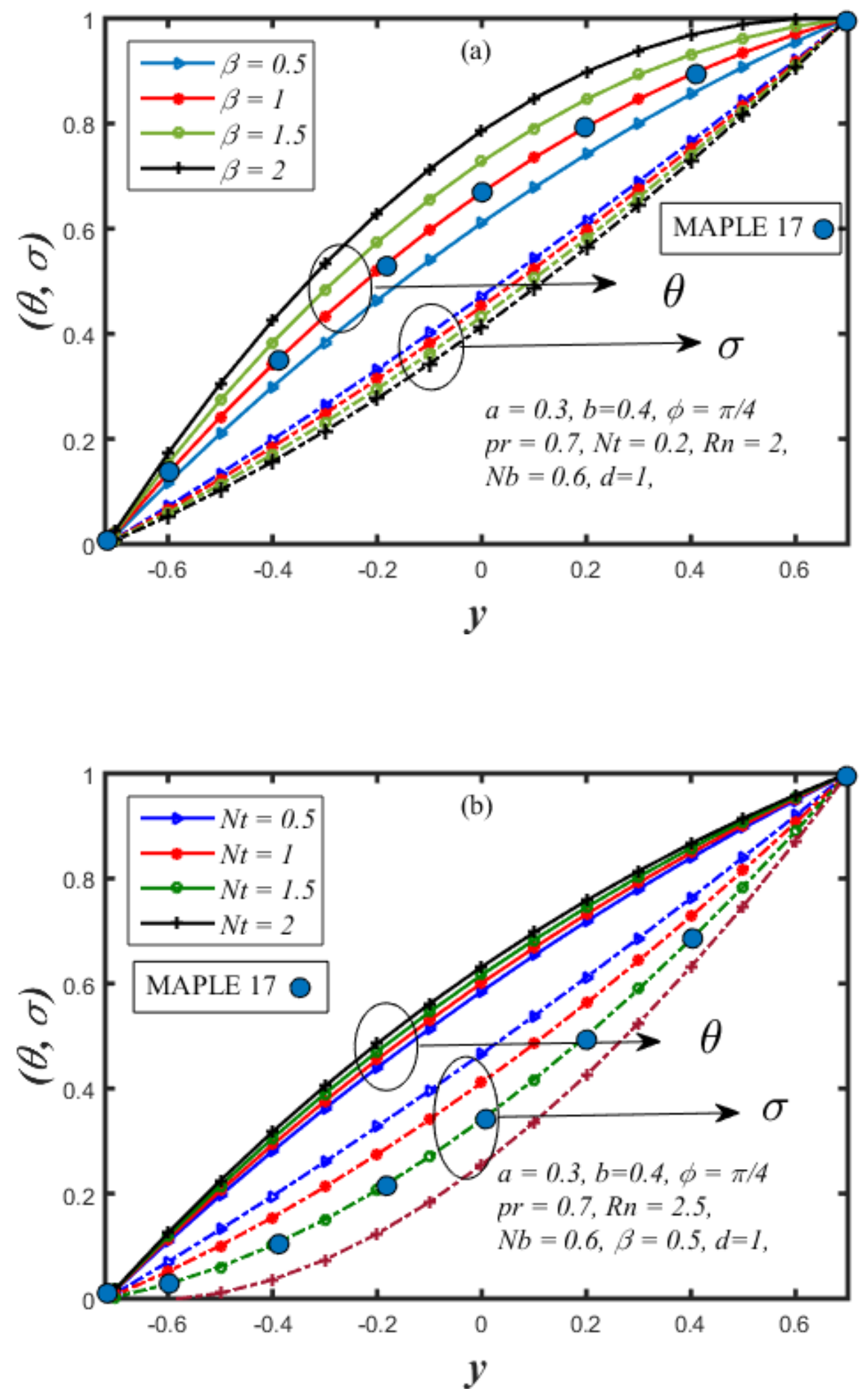

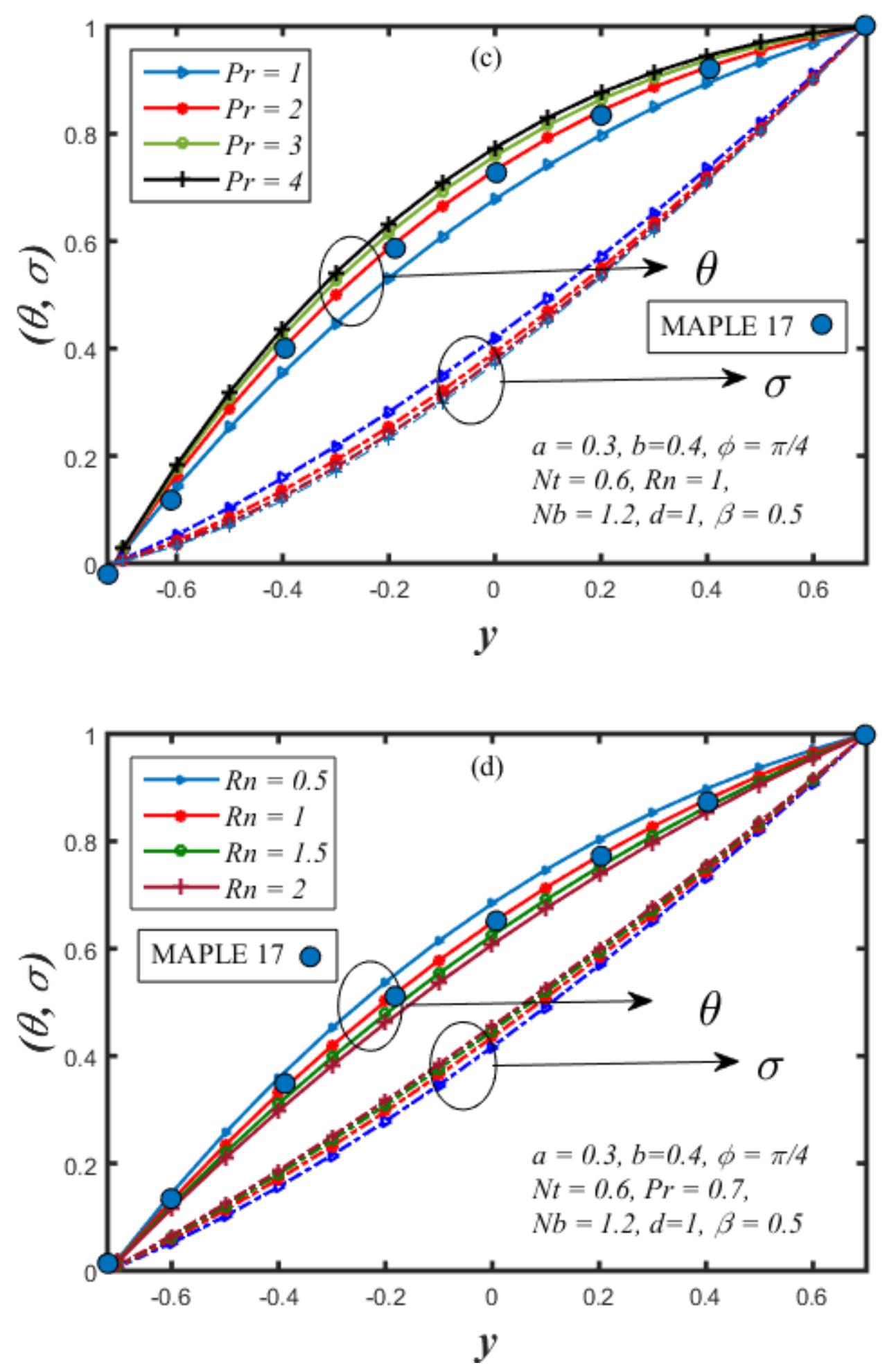

Figure 4 Temperature and nanoparticle volume fraction profiles with variation in (a) $\beta$, (b) $N t$, (c) Pr, (d) Rn . 


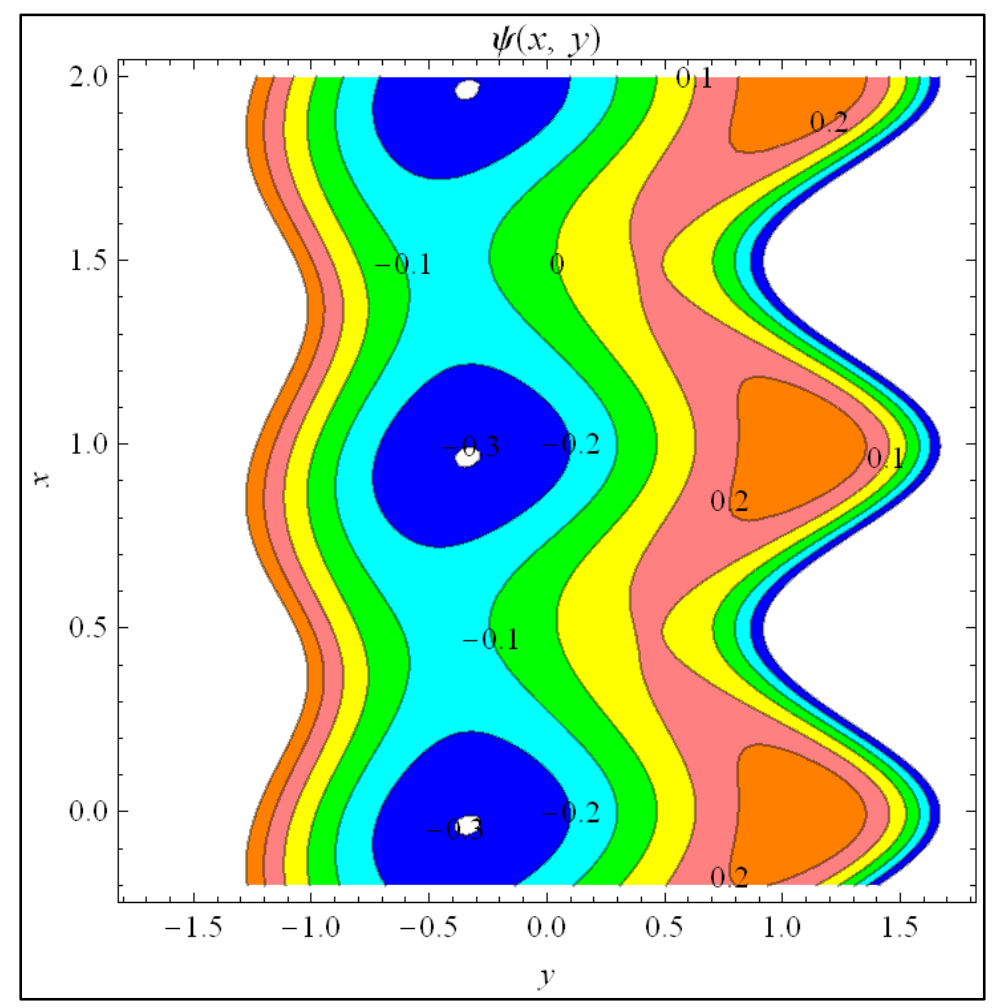

(a)

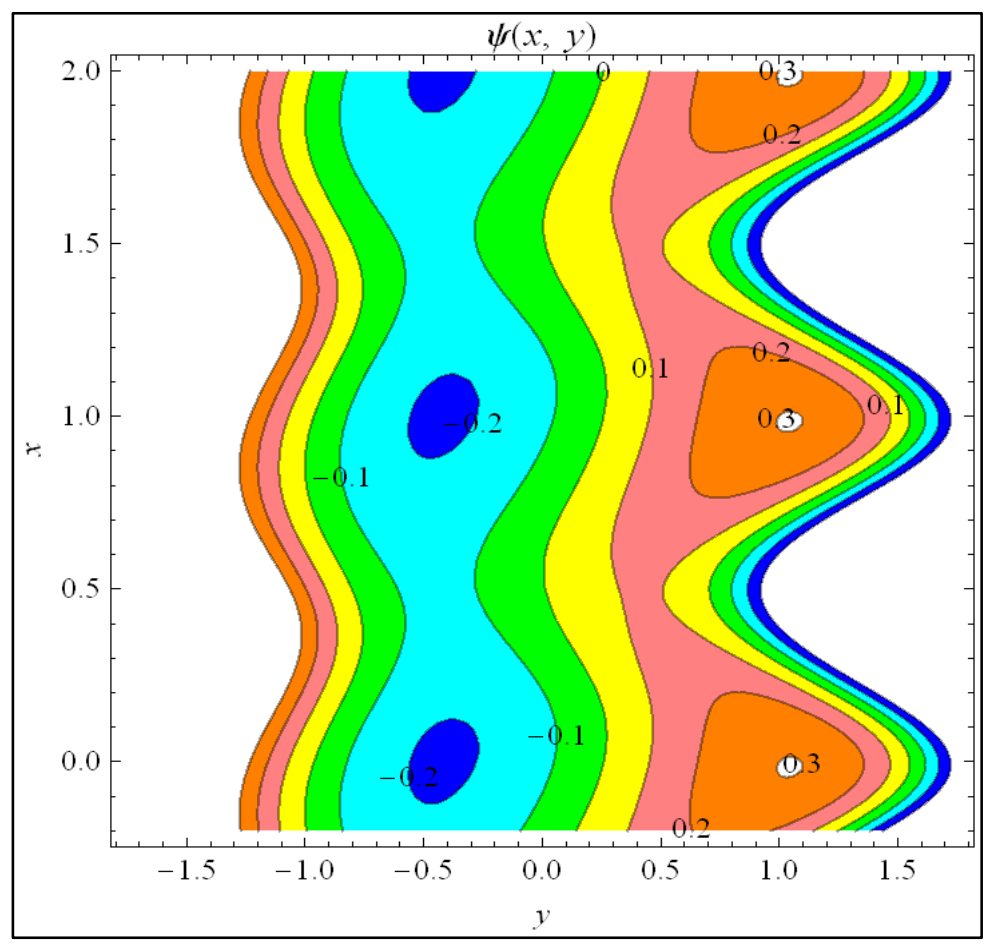

(b)

Fig.5. Stream lines at $a=0.3, b=0.2, \operatorname{Pr}=0.7, R n=0.5, \beta=0.5 N b=0.5, N t=0.5, M=1$, $d=1.1, B r=3, \Theta=2, K=0.2, \phi=\pi / 4, G r=2, \operatorname{Pr}=2, N t=1$, for (a) $\alpha=0$, (b) $\alpha=0.1$. 


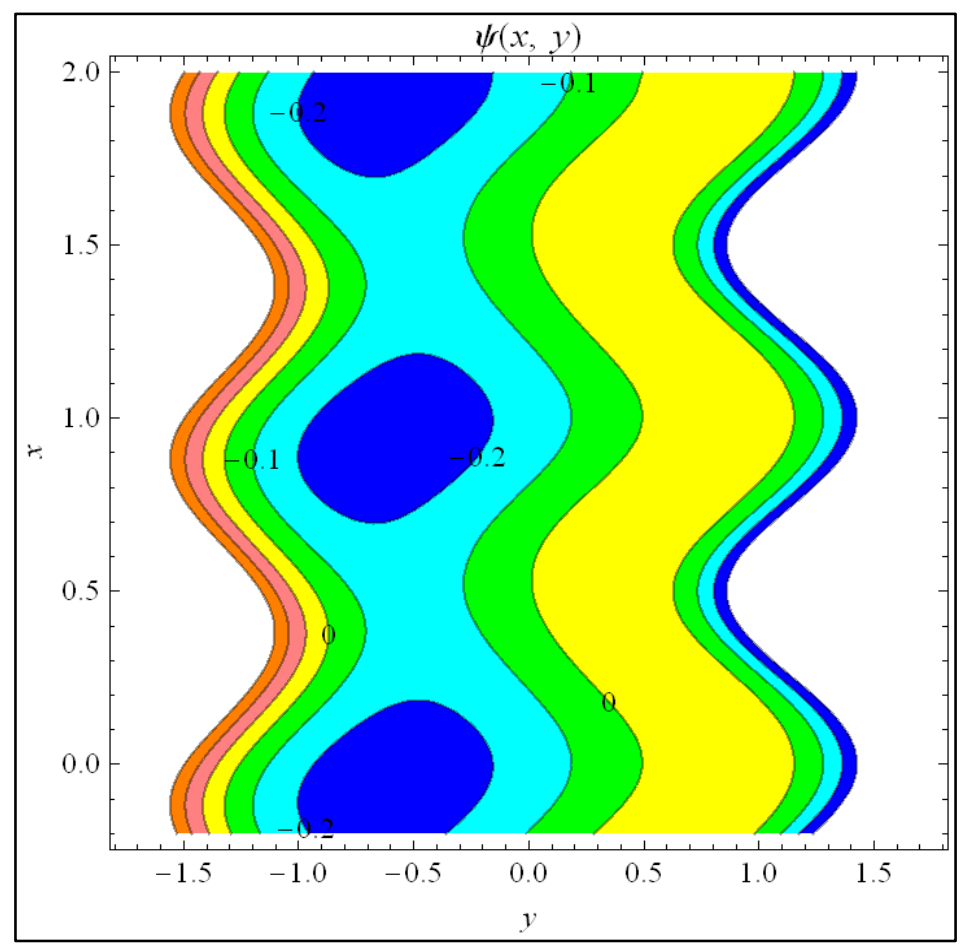

(a)

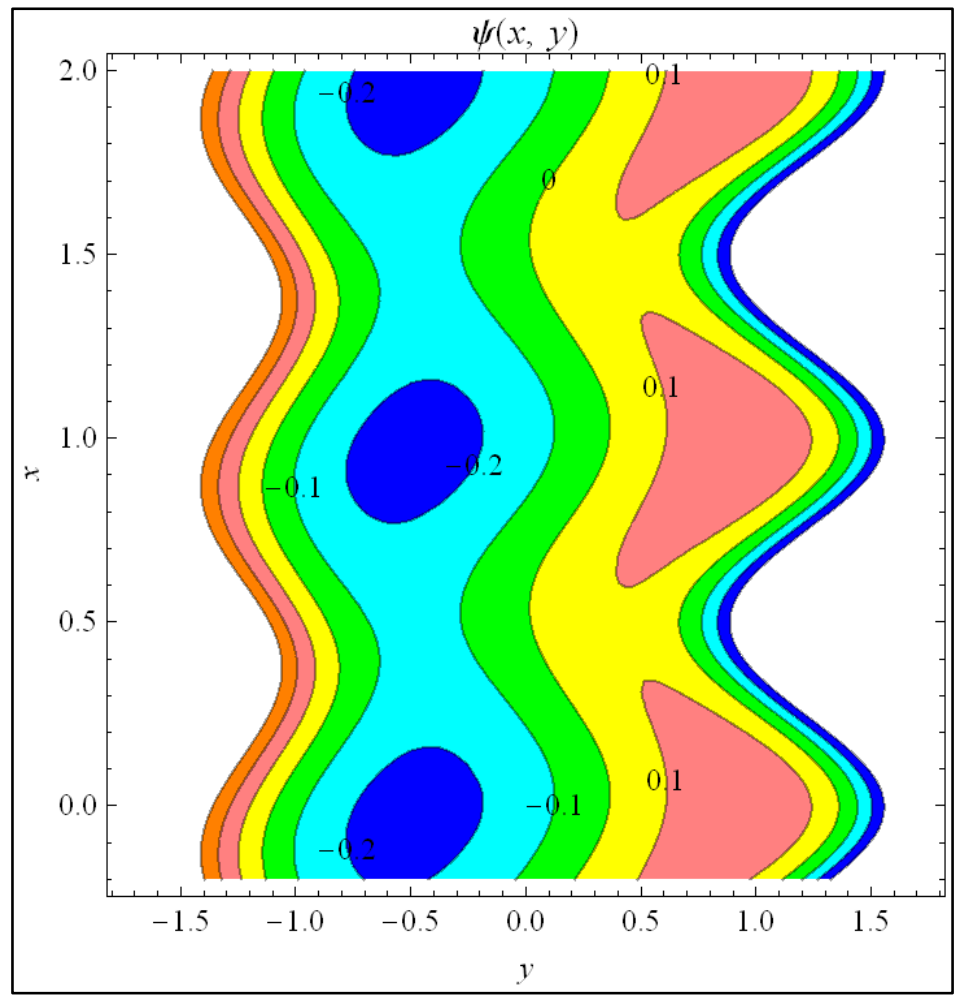

(b)

Fig.6. Stream lines at $a=0.3, b=0.2, \operatorname{Pr}=0.7, R n=0.5, \alpha=0, N b=0.5, N t=0.5, M=1$, $d=1.1, B r=3, \Theta=2, K=0.2, \phi=\pi / 4, G r=2, \operatorname{Pr}=2, N t=1$, for (a) $\beta=0$ (b) $\beta=0.5$. 


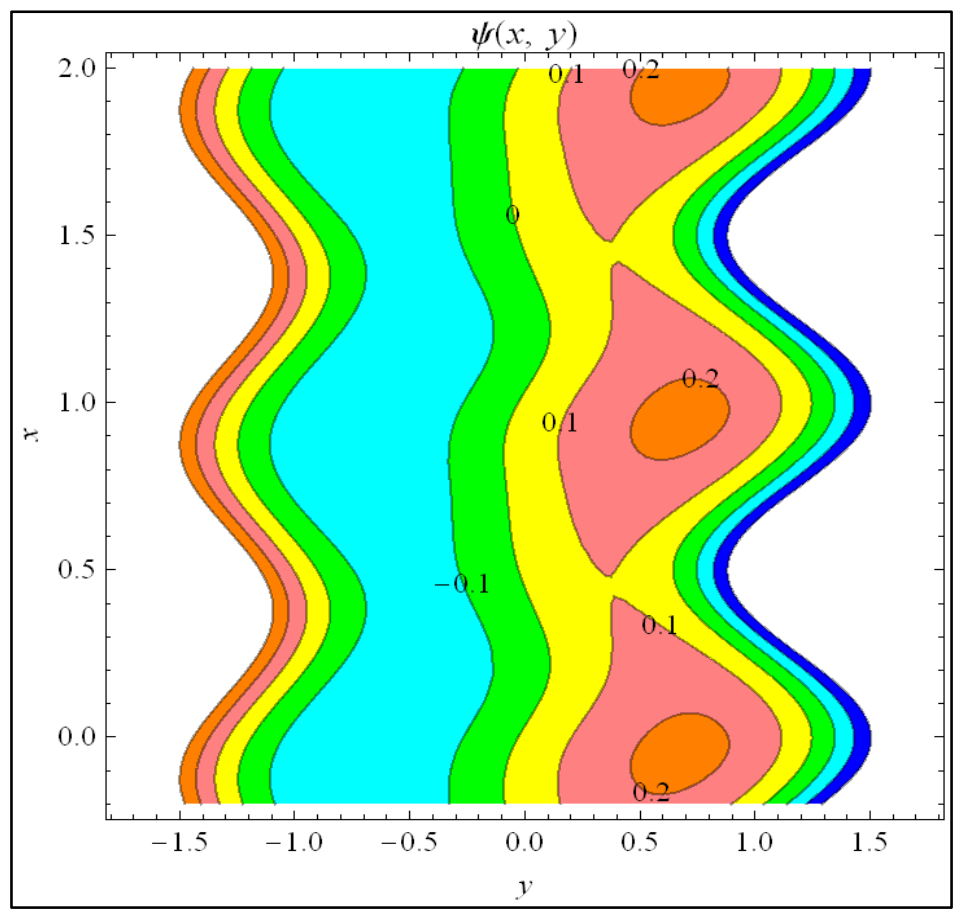

(a)

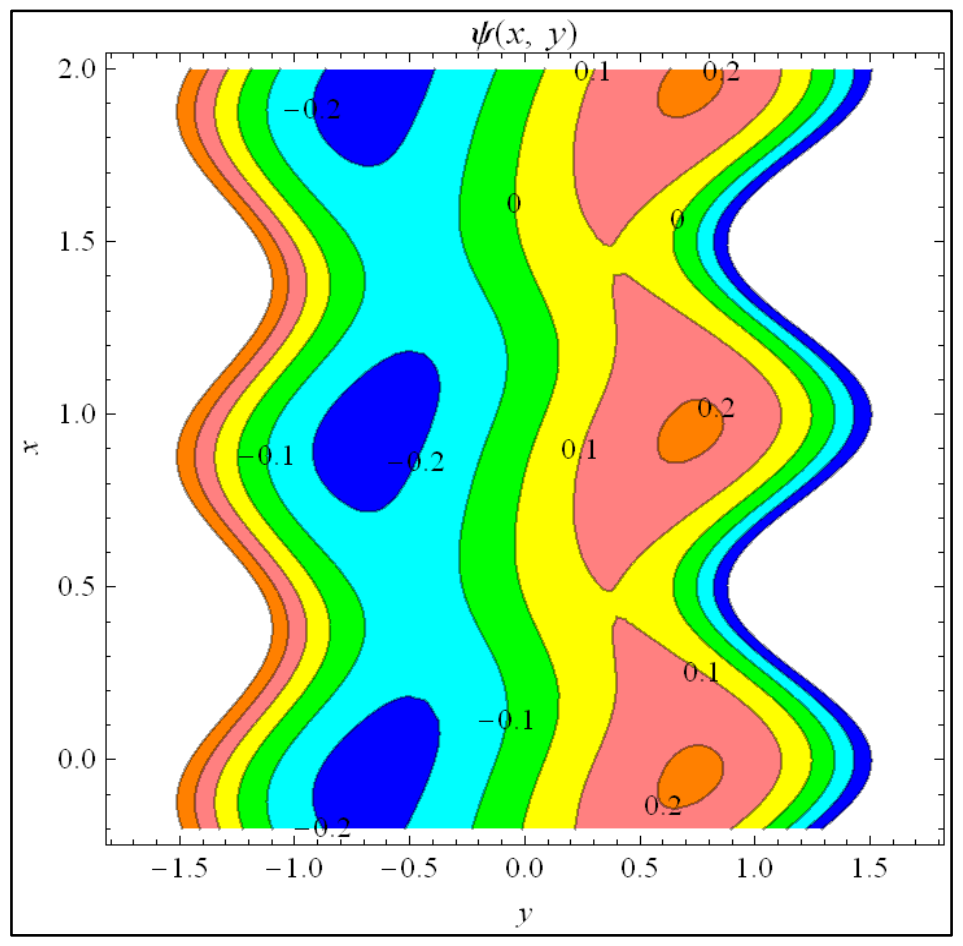

(b)

Fig.7. Stream lines at $a=0.3, b=0.2, \mathrm{Pr}=0.7, R n=0.5, \alpha=0, N b=0.5, N t=0.5, \beta=0.25$, $d=1.1, B r=3, \Theta=2, K=0.2, \phi=\pi / 4, G r=2, \operatorname{Pr}=2, N t=1$, for (a) $M=0$, (b) $M=1$. 


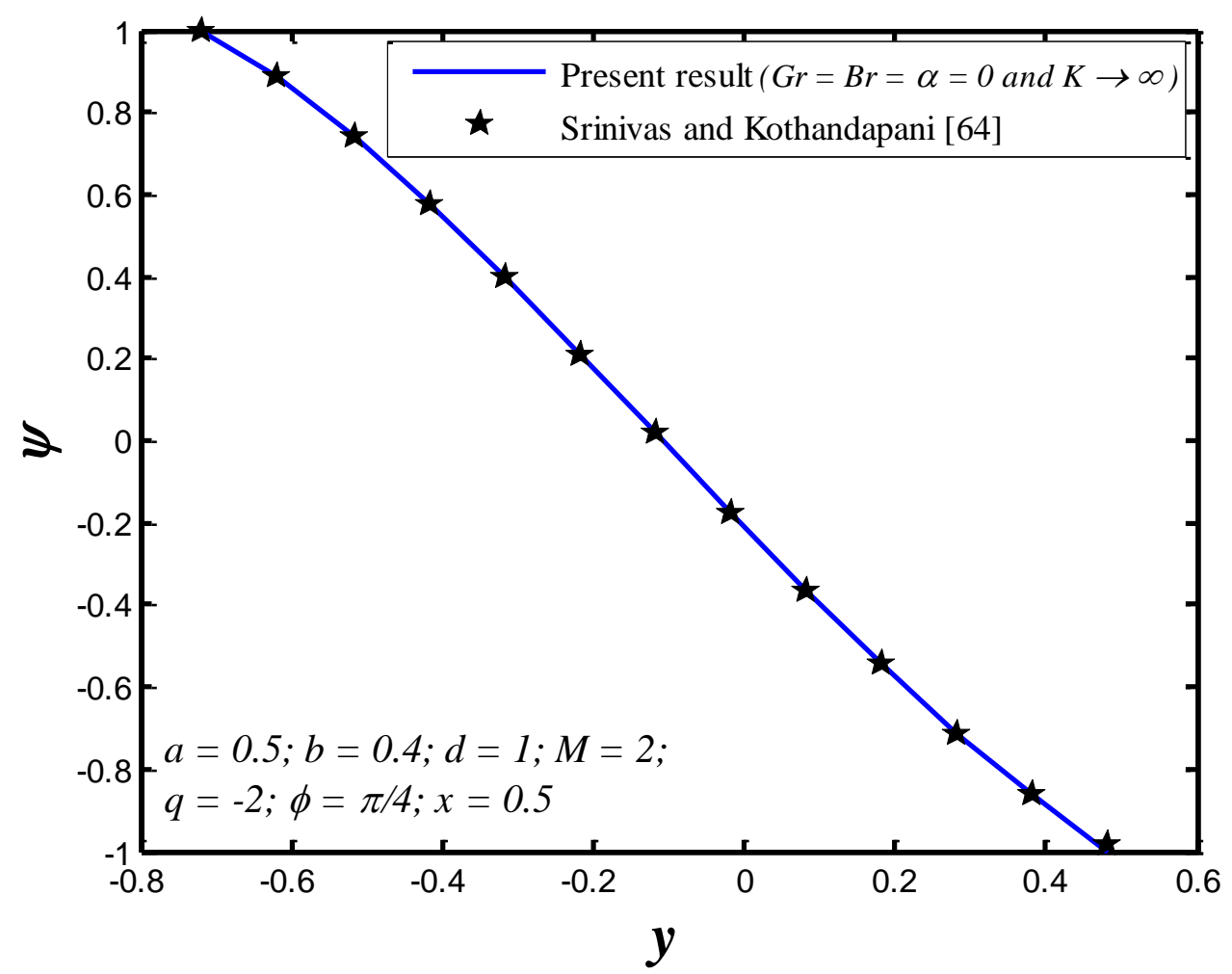

Figure 8. Comparison result of stream function versus transverse axis.

\subsection{Pumping characteristics}

The pressure rise per wavelength can be calculated by using the form $\Delta p=\int_{0}^{1}\left(\frac{\partial p}{\partial x}\right) d x$. The pressure rise is computed numerically using the symbolic software Mathematica for the perturbation solutions and MAPLE17 for the Runge-Kutta-Merson numerical solutions. The pressure rise $(\Delta p)$ versus mean flow rate $(\Theta)$ for various values of $\alpha, M, K, R n, \beta$ and $B r$ is illustrated in Figs. 2 (a-f). Fig.2(a) portrays that the influence of Reynolds viscosity parameter on the pressure rise versus the mean flow rate. One can insure that the pressure rise increases with increasing Reynolds viscosity parameter $(\alpha)$ for the pumping region $(\Delta p>0, \Theta>0)$. It is also evident that volumetric flow rate is elevated for Newtonian nanofluid $(\alpha=0)$ and depressed for nonNewtonian nanofluid i.e. for non-zero Reynolds viscosity parameter $(\alpha>0)$. Additionally, peristaltic pumping is inhibited with increasing temperature-dependence of the nanofluid 
viscosity. This is also consistent with the simultaneous deceleration in the axial flow with greater nanofluid viscosity. Pressure and velocity (linked to flow rate) respond in opposite ways in hydrodynamics- the inverse relationship is clearly demonstrated in all the negative gradients in the profiles in the plot. Evidently pressure rise is minimized with the Newtonian nanofluid (least viscosity) and maximized with strongly non-Newtonian nanofluid (highest viscosity). The implication for solar pump performance is that if greater pressures are required (as opposed to flow acceleration), this may be achieved via increased doping of the working fluid with nano-particles.

Choi [11] has highlighted the strong modification in nanofluid rheology with increased concentrations of nano-particles. The change in viscosity is intimately related to heat transfer enhancement of nanofluids in particular when buoyancy forces are present, as in the present study, as will be elaborated in due course. Thus viscosity variation as studied by the Reynolds model may provide a good insight into the improved efficiencies reported in solar energy systems doped with nanofluids at higher temperatures as noted by Cingarapu et al. [17], Alashkar and Gadalla [20]. Furthermore, since magnetic pumping is also present it is noteworthy that the present computations are consistent with the earlier simulations of Shahidian et al. [26] where rheological and higher viscosity was observed to also generate pressure difference elevation in pump operations. In Fig. 2(b), we examine the effect of Hartmann number $(M)$ on the pressure rise verse mean flow rate. Clearly pressure rise diminishes with growing values of Hartmann number for the peristaltic pumping region $(\Delta p>0, \Theta>0)$. The Lorentz magnetic drag force i.e. $-M^{2} \partial^{2} \psi / \partial y^{2}$ in eqn. (21) is generated by the application of magnetic field in the $\mathrm{Y}$-direction (transverse to the axial velocity direction i.e. the $\mathrm{X}$-direction). This accelerates the flow and concurrently depletes the pressure rise in the solar magnetic pump channel. The profiles are all therefore linear decays i.e. with maximum pressure rise there is a corresponding minimal flow rate and vice versa. This concurs with the findings of Lim and Choi [21] for the similar scenario of a two-dimensional channel with sidewalled electrodes, although in our study wall deformability is present i.e. the channel walls propagate peristaltic waves (we have set the amplitudes of left and right walls i.e. $a, b$ as 0.3 and 0.2 respectively and also the phase difference is prescribed as $\pi / 4)$. Similar observations have also been reported by Das et al. [22], Ho [23] and Leboucher et al. [24]. These studies have also shown in agreement with our computations that pressure rise in magnetohydrodynamic pumping is lowest with strong magnetic field $(M=0.5)$ and highest with absence of magnetic field i.e. electrically- 
non-conducting nanofluid $(M=0)$. These trends are sustained at all values of volumetric flow rate $(\Theta)$. Fig. 2(c) depicts the influence of permeability parameter $(K)$ on the pressure rise versus mean flow rate distributions. From this figure, we discern that an increase in the permeability parameter initially depresses the pressure difference; however, with subsequent increase in volumetric flow rate the pattern is strongly reversed with a significant upsurge in pressure difference during peristaltic pumping. Permeability is a key characteristic of porous media. This parameter characterizes the hydrauic transmissivity of the porous medium. It does not however relate to the porosity of the medium which is connected to the relative volume of voids to the volume of solids. Permeability features in the linear Darcian impedance term in the reduced momentum equation (21), namely the term $-(1 / K) \partial^{2} \psi / \partial y^{2}$ and this is associated with the retarding effect of the porous solar wafer fibers. Darcy's law is generally valid for Reynolds numbers less than 10 i.e. it is applicable for viscous-dominated pumping dynamics as opposed to inertially-dominated transport (this requires a Forchheimer-extended Darcy model to simulate second or higher order drag effects and is presently being considered as an extension to the current work to extend the operational range to higher Reynolds numbers). With increasing permeability, the regime solid fibers progressively decrease. This results in effective enhancement in pressure differences across the channel length i.e. a boost in $\Delta p$. This behavior is sustained for the majority of positive volumetric flow rates. The initial depletion in pressure rise is more associated with the entry length hydrodynamics and negative flow rates (back flow) and a threshold volumetric flow rate is necessary to enforce the dominant effect of permeability as elucidated in detail by Wang et al. [25] and also earlier by Nimr and Alkam [38] for different solar cell and pump configurations i.e. the permeability effect is independent of conduit geometry and is controlled by the nature of the wafer material. The presence of a low permeability porous medium (solar wafer) i.e. $K=0.1$ generally damps the pressure difference generated in the pump whereas higher permeability i.e. $K=0.3$ induces the opposite effect. It is also pertinent to note that the permeabilities studied are high i.e. the medium is sparsely packed which obviates the possibility of compressibility effects, consolidation effects or compaction phenomena, as further highlighted by Ren et al. [36]. Fig. 2(d) presents the impact of thermal radiation flux on pressure rise versus mean flow rate profiles. It is remarked that there is a strong presence of positive pumping $(\Theta>0$ and $\Delta p>0)$ when $R n=0$ as compared with $R n>0$. Thermal radiation is the most significant contribution from solar heat 
loading [1]. The parameter $R n$ arises in the augmented energy equation (19) i.e. in the term $R n$ $\partial^{2} \theta / \partial y^{2}$

Rosseland's model assumes radiative equilibrium and that the nanofluid has gray properties which are popular in solar pump designs. Furthermore, Rosseland's model assumes that the intensity is the black-body intensity at the nanofluid temperature and since it is generally confined to incompressible flows it is particularly appropriate for low speed transport characteristic of porous media hydromagnetic pumping. The parameter $R n$ embodies the relative contribution of conduction heat transfer to thermal radiation transfer. It is variously known in thermal physics as the Stark number and Rosseland-Boltzmann number [1]. Large $R n$ values imply small radiation contribution and small $R n$ values correspond to high radiative flux. As $R n \rightarrow 0$, thermal radiation flux contribution vanishes and the dominant mode of heat transfer is thermal conduction. Hence with smaller $R n$ values, thermal radiation is stronger than thermal conduction (the contribution is only equal for both modes of heat transfer when $R n=1$ ). Thermal radiation supplements the fluid thermal conductivity via the energy equation and serves to increase temperatures, simultaneously reducing momentum transfer which serves to decrease pressure difference at positive flow rates (the converse effect is induced at negative flow rates). The influence of radiative flux on velocity, temperature and nano-particle volume fraction distributions is described in due course. The variation in pressure rise for different values of heat source/sink parameter $(\beta)$ is illustrated in Fig. 2(e). It is evident that pressure rise is assisted with an increase in heat source (generation) parameter in all the peristaltic pumping regions. The maximum peristaltic pumping is achieved when $\beta>0$ as compared with the least efficient pumping attained with $\beta=0$. Generally, the presence of heat generation (mimicking for example thermal hot spot zones in solar pumps) strongly elevates temperatures (see fig 4a). This also assists in momentum development and manifests in pressure difference depletion. The influence is maintained at both negative and positive flow rates i.e. whether correct pumping or reverse flow is present. The observations are consistent with the findings of Tien and Vafai [39]. The heat source effect is also particularly relevant to ground-heat source solar pumps [33]. It works effectively to energize the pumping fluid and aids in increasing thermal pumping efficiency. Figs. 2(f) display the influence of the species buoyancy force (simulated via local nanoparticle i.e. solutal Grashof number $B r$ ) on the pressure rise $(\Delta p)$ versus mean flow rate $(\Theta)$. Br arises in the species buoyancy term in the reduced momentum eqn. (21) i.e. Bro and this term couples the momentum field with the nano-particle 
species concentration field described by eqn. (20). It represents the relative magnitude of the species buoyancy force to the viscous hydrodynamic force in the channel. When $\mathrm{Br} \rightarrow 0$ species (solutal) buoyancy effects vanish. Thermal Grashof number $(G r)$ is prescribed a value of 2 implying the thermal buoyancy force is double the magnitude of the viscous hydrodynamic force. Pressure rise is considerably elevated with increasing solutal Grashof number $\mathrm{Br}$. This applies at both negative and positive flow rates and in the pumping region $(\Delta p>0)$, the augmented pumping region $(\Delta p<0)$, and the free pumping region $(\Delta p=0)$. Pressure rise is clearly suppressed with absence of species buoyancy forces indicating that the presence of nano-particles is beneficial to solar peristaltic pump performance. Nano-particle solutal buoyancy is shown therefore to be a critical characteristic which modifies the pump hydrodynamic regime. It is also judicious to mention that positive values of $\mathrm{Br}$ correspond to nano-particle transfer from the channel walls by natural species convection currents which implies a reduction in wall mass transfer rate (channel wall nano-particle concentration gradient).

\subsection{Axial Velocity distribution}

The effects of the principal flow parameters i.e. Hartmann magnetic number, thermal conductionradiation parameter (Stark number), Prandtl number, permeability parameter, heat source parameter and Reynolds viscosity parameter on axial velocity distribution $\left(u=u_{0}+\alpha u_{1}\right)$ are visualized in Fig. 3 (a-f) at the axial location $x=0.5$. It is observed that the axial velocity distributions exhibit a parabolic nature in which the maximum/minimum axial velocity appears at the core zone of the channel. However, there is a slight dis-symmetry which is a manifestation of the different amplitudes of left and right walls i.e. $a, b$ as 0.3 and 0.2 respectively i.e. the profiles are either skewed to the left or to the right wall. The influence of Hartmann number $(M)$ on axial velocity distribution versus transverse coordinate is plotted in Fig.3 (a). It is noticed that the performance of axial velocity near the channel walls and at the core part of the channel demonstrates a reflective symmetry approximately about the channel centre line $(y=0)$. Axial velocity enhances with an increase in Hartmann number near the lower deformable channel wall. However, it reduces in the core region of the porous medium channel and all profiles merge towards the upper wall i.e. there is invariance in the magnetic field effect near the upper wall. The presence of magnetic field in the electrically-conducting magnetized nanofluid, as explained earlier, mobilizes the Lorentz magnetic force. This acts to resist the channel flow in magnetic 
generator operations but accelerates the flow in magnetic pumping operations (as considered here). Fig.3 (b) shows the effect of thermal radiation on axial velocity distribution. It is noticed that velocity is depressed in the lower channel half space i.e. $y \in[-1.07,-0.2499]$ whereas it is enhanced in the upper channel half space, with increasing $R n$ i.e. with decreasing thermal radiative flux. Energization of the nanofluid with stronger solar radiative flux therefore decelerates the pumping flow. The Prandtl number effect on the axial velocity distribution is plotted in Fig.3(c). It is apparent that the axial velocity is raised with an elevation in Prandtl number over the lower channel half-space range $y \in[-1.07,-0.2386]$; however, in the upper channel half space range velocity is strongly decreased with increasing Prandtl number. The no-slip condition requires that the flow velocity at the surface of the channel walls is zero and that the nanofluid temperature is equal to the surface temperature. Prandtl number defines the relative rate of momentum diffusion to energy (heat) diffusion in the regime. For Prandtl number of unity, both heat and momentum diffuse at the same rate. In the entry length zone of the magnetohydrodynamic solar pump channel, for Prandtl number below unity, the thermal boundary layer is thicker than the velocity boundary layer. The flow is also accelerated with increasing Prandtl number in the lower channel half space since this is closer to the entry length zone whereas the converse effect is induced in the upper channel half space. As elaborated in Shahidian et al. [26] it is unfeasible to achieve a consistent acceleration or deceleration throughout the entire magnetohydrodynamic pump with purely an increase in Prandtl number. Fig.3 (d) displays the axial velocity distribution for three different value of the permeability parameter $(K=0.1,0.2$ and 1$)$. It is viewed that the axial velocity is suppressed in the region $y \in[-1.07,-0.2846$, whereas it is magnified in the region $y \in[-0.2846,0.64]$. The switch-over for this behavior is located to the left of the channel centre line. Maximum axial flow is generated mid-way within the upper channel half space with maximum permeability i.e. minimal Darcian retarding force. Increasing permeability parameter results in depletion in resistance of the solid fibers to the percolating nanofluid. This accelerates the nanofluid pumping and increases the shearing effect at the channel walls leading to greater shearing stresses at these boundaries. As $K \rightarrow \infty$ the porous matrix disappears and the regime becomes pure nanofluid. In the opposite limit as $K \rightarrow 0$, the medium permeability vanishes and the pump is completely filled with solid material preventing pumping operations. Many studies including Vasiliev et al. [37] and Ramesh [54] have confirmed that optimum solar and peristaltic 
pump performance and flow control is attainable with high but not excessive permeabilities. Fig. 3 (e) shows the influence of the three different values of heat source parameter $(\beta=0,0.5$ and 1$)$ on the axial velocity distribution for the fixed values of other parameters. It is evident that with increasing heat source/sink parameter, $\beta$, the axial velocity field is depleted in the lower channel half space $y \in[-1.07,-0.4046]$, where as it is enhanced in the upper channel half space in the range $y \in-[-0.4046,0.64]$. The influence of the Reynolds viscosity $(\alpha)$ parameter on $u$ is presented in Fig. 3(f). Axial velocity distribution $(u)$ reduces with the increase in Reynolds viscosity effect $(\alpha)$ in the lower channel half space (here the boost in viscosity inhibits strongly the entry flow in the solar pump channel duct) whereas the contrary behavior is computed in the upper channel half space (right hand side of the channel geometry). This trend is consistent with several studies on nanofluid non-Newtonian viscosity behavior, including Choi [11], and Shahidian et al. $[26,28]$. The latter investigation has confirmed experimentally that viscosity abnormally increases when an increase in volume concentration of nano-particles is present and this leads to deceleration in flows earlier in micro-channels with subsequent acceleration.

\subsection{Temperature and Nanoparticle volume fraction distributions}

In Figs.4 (a-d) the evolution of nanofluid temperature distribution $(\theta)$ and nanoparticle volume fraction $(\sigma)$ with respectively different values of heat source parameter $(\beta)$, thermophoretic nanoscale parameter $(N t)$, Prandtl number (Pr) and conduction-radiation Stark number $(R n)$. A significant feature of the temperature distributions is the concave upward profile whereas a downward concave topology is exhibited by the nanoparticle volume fraction distributions. The heat source parameter influences on temperature and nanoparticle fraction distributions are presented in Fig. 4(a). It is evident that the temperature increases with enhancing the heat source parameter effects (since thermal energy is imparted to the nanofluid with heat source in the pump) whereas the nanoparticle volume fraction (i.e. nano-particle concentration magnitude) is clearly reduced with increasing the heat source parameter. The implication is that the heat generation effect only assists thermal distribution homogeneity in the channel whereas it is counter-productive for the diffusion of nano-particles. The temperature and nanoparticle volume fraction distributions for the various values of thermophoresis parameter $(N t)$ are presented in Fig.4 (b). Inspection of the graph reveals that the temperature distribution increases with an increase in the thermophoresis 
parameter. It also noticed from this figure that the nanoparticle volume fraction reduces with an increase in the thermophoresis parameter. Different nanoparticles types transport differently under the thermophoretic force which is associated with migration of nano-particles from hot to cold surfaces. This is of great utility in solar magnetic nanofluid pumps [26-28] since via selection of specific nano-particles, customized performance may be achieved. It is further of interest to consider the mechanism of thermal enhancement in nanofluids under solar radiative conditions. It is also noted that the Brownian motion parameter $(\mathrm{Nb})$ is fixed at 0.6. Since Brownian dynamics is also present, the nanofluid molecules are organized into an ordered layer at the nano-particle solid-liquid interface which results in thermal conductivity in that ordered layer being lower than thermal conductivity of the solid particles but larger than that of the base fluid. As earlier suggested by Buongiorno [13], this interfacial layer is a solid-like structure, and it is referred to as nanolayer. This hypothetical nanometre size layer is considered as a thermal layer between the solid particle surface and the base fluid and to the current state of knowledge of nanofluid mechanics, this nanolayer is one of the most probable mechanisms producing the popular thermal conductivity enhancement. Infact the existence of even a thin nanolayer at relatively high Brownian motion parameter values still may contribute markedly to the elevation in thermal conductivity of nanofluids, mainly when the particle diameter is smaller than $10 \mathrm{~nm}$. In conjunction with thermophoresis, the extra energy transport of nanoparticles is due to result of Brownian motion. The relative motion between nanoparticles and base fluid molecules generates micro-convection which when summated over the entire body of the nanofluid results in an effective boost in heat transfer i.e. temperatures. The present theoretical results confirm these observations. However, it is sincerely hoped that experimental solar engineering researchers will be motivated to verify actual performance in both laboratory models and scale-up processes to actual implementation of the current solar magnetic biomimetic nanofluid pump configuration. The influences of four different value of Prandtl number (i.e., $\operatorname{Pr}=1,2,3$ and 4) on temperature and nanoparticle volume fraction are computed in Figs. 4(c). In is found that elevation in Prandtl number leads to a strong enhancement in fluid temperature whereas it depletes the nanoparticle volume fraction. Generally, higher Pr nanofluids will have relatively low thermal conductivities which will suppress thermal conduction heat transfer from the wall and reduce thermal boundary layer thickness, resulting in higher nanofluid temperatures throughout the channel space. Smaller values of Pr are equivalent to increasing thermal conductivities, and therefore heat is able to diffuse 
away from the deformable pump walls more rapidly than for higher values of Pr. Hence in the case of smaller Pr the boundary layer is thicker and the rate of heat transfer to the wall is reduced. This has important implications in practical solar pump operations. In Fig. 4(d), we observe that with increasing thermal radiation (lower $R n$ values) there is a substantial elevation in fluid temperature. It is also noticed that the thermal radiation effect suppresses nanoparticle volume fraction magnitudes i.e. counter-acts diffusion of nano-particles, which has been confirmed in many experimental investigations including Said et al. [40] and Du and Tang [41].

\subsection{Trapping phenomena}

A unique characteristic of the present solar pump design is the peristaltic wave feature achieved with distensible channel walls. This generates an intriguing phenomenon known as trapping, the formulation of an inside circulating bolus of nanofluid which migrates together with the wave at a fixed mean flow rate. In axisymmetric peristaltic flows, the positive motion displacing fluid forward manifests in a torus shape. The bolus is trapped by the wave and therefore propagates forward with the same speed as that of the wave. However, in the current analysis, non-symmetric peristaltic pumping is studied owing to the difference in amplitudes of left and right walls i.e. $a, b$ as 0.3 and 0.2 respectively. However, a single phase difference of $\pi / 4$ between the left and right wall peristaltic waves is assumed. Although asymmetric peristaltic flows may generate reflux (or retrograde flux), that is, reversed motion of fluid in the opposite direction opposite to the net flow (i.e. in the negative $\mathrm{x}$-direction) this has not been observed in the current work. The streamlines for different Reynolds viscosity parameter $(\alpha)$, heat source/sink parameter $(\beta)$ and Hartmann magnetic number $(M)$ for fixed values of other parameters are illustrated in Figs.5-7. Fig. 5(a-b) indicate that with increasing Reynolds number viscosity $(\alpha)$ the size of trapped bolus increases in amplitude in the right part (upper half space) of the channel and the reverse situation is generated in the left part (lower half space) of the channel wall. The impact of two different values for heat source parameter ( $\beta=0$ and $\beta=0.5$ ) on trapping is shown in Fig. 6(a-b). One can notice that the size of the bolus increases in the vicinity of the right part of the channel with increasing heat source parameter. Fig. 7(a-b) clearly reveals that the size of tapped bolus increases with magnetic field

effect i.e. as Hartmann number increases from the non-magnetic case $(M=0)$ to the strongly magnetic case $(M=1)$ trapping is more potent in the channel regime. 


\subsection{Further Validation with Published Literature}

Fig.8 is explained to authenticate the results of proposed model without buoyance forces $(B r=G r=0)$, Permeability parameter $(K)$ and Reynolds number viscosity parameter $(\alpha)$ with existing results Srinivas and Kothandapani [64] in the asymmetric channel. It is perceived that the results of proposed model is significantly close to that described by Srinivas and Kothandapani [64] in the entire width of the channel.

\section{CONCLUDING REMARKS}

A theoretical study has been presented to simulate the influence of temperature-dependent viscosity, thermal radiation flux, nanoscale phenomena, heat generation, solutal (nano-particle) buoyancy force and magnetic field on the two-dimensional flow, heat and species diffusion in a solar biomimetic (peristaltic) magnetohydrodynamic nanofluid pump containing a homogenous, isotropic porous medium. A modified Buongiorno [13] nanofluid model is deployed which emphasizes thermophoretic body force and Brownian dynamic effects. To simulate solar thermal loading conditions the Rosseland radiative diffusion flux model is implemented and heat generation is included. Multiple amplitudes and phases are considered for the deformable channel walls. The moving boundary value problem is non-dimensionalised and perturbation solutions are derived for axial velocity, temperature and nanoparticle volume fraction. Validation is conducted with Maple numerical quadrature and volumetric flow rate, pressure difference and streamline distributions are also computed. The impact of Reynolds number viscosity $(\alpha)$, heat source/sink parameter $(\beta)$, Prandtl number $(\operatorname{Pr})$, Hartmann number $(M)$, thermal radiation $(R n)$, permeability parameter $(K)$ and local nanoparticle Grashof number $(B r)$ on thermal and flow characteristics is visualized. The important findings in the current study may be summarized as follows:

$>$ The free pumping flux for a fluid with variable viscosity is greater than that for a fluid with constant viscosity.

$>$ Greater peristaltic work has to be expended versus a greater pressure for a fluid with constant viscosity when compared with that fluid of variable viscosity.

$>$ Pressure rise increases as the Reynolds viscosity of nanofluid and the heat generation effects are increased. 
Fluid axial velocity distribution is only enhanced with Reynolds viscosity increasing, in the inner core zone of pump channel, owing to the lesser impact of shear stress (wall friction) in the inner core.

The nanoparticle fraction distribution exhibits the reverse behavior as compared with the temperature distribution under the influence of thermophoresis, Reynolds viscosity, Prandtl number and magnetohydrodynamic Hartmann number.

$>$ With increasing Reynolds viscosity, the size of trapped bolus decreases in amplitude in the left section of the channel whereas the opposite behavior is observed in the right channel half space.

$>$ Increasing radiation solar flux significantly heats the nanofluid whereas it inhibits nano-particle diffusion in the regime.

$>$ Increasing magnetic Hartmann number serves to reduce pressure differences in the pumping channel with a corresponding acceleration in the axial flow.

$>$ Excellent accuracy, stability and convergence is achieved with both the perturbation solutions and MAPLE17 computations.

$>$ Pressure difference is strongly boosted with increasing solutal Grashof number at both negative and positive flow rates and in all three solar peristaltic pumping regimes i.e. the pumping region $(\Delta p>0)$, the augmented pumping region $(\Delta p<0)$, and the free pumping region $(\Delta p=0)$.

> Solar thermal radiation flux energizes the nanofluid in the pump and elevates temperatures via the augmentation of the nanofluid thermal conductivity; however, increasing radiation flux acts to reduce pressure difference at positive flow rates (the reverse trend is computed at negative flow rates).

The presence of a low permeability porous medium (solar wafer) is observed to strongly decelerate axial flow and suppress pressure difference generated in the pump whereas higher permeability manifests in flow acceleration and pressure difference elevation.

At all values of Pressure rise in magnetohydrodynamic pumping is stifled with strong magnetic field whereas it is enhanced for electrically-non-conducting nanofluids (i.e. when the external magnetic field in the pump circuit is switched off). 
The present study constitutes a first attempt to simulate a novel hybrid solar magnetohydrodynamic nanofluid micro-pump for implementation in astronautics, biomedical systems and environmentally-sustainable energy generation. Promising results have been obtained. However, the current work has been confined to two-dimensional optically-dense nanofluids. Future work will consider three-dimensional numerical simulations with ANSYS CFD software and furthermore will explicitly explore the impact of more complex thermal radiation effects. In this regard, optical thickness and absorption coefficient are key aspects to study since they quantify how much a given medium retards the passage of thermal radiation. Radiative intensity falls by an exponential factor when optical thickness is unity. Physically optical thickness will be a function of the absorption coefficient, medium density and propagation distance in actual solar pumps. It may be advantageous to explore the $\mathrm{P} 1$ radiative flux model which although more computationally expensive predicts the actual influence of solar radiative flux more accurately than the Rosseland model and also is applicable to non-gray nanofluids although it may slightly over-predict radiative fluxes from localized heat sources or sinks. Non-Darcy porous media effects are also being considered in order to extend the operational Reynolds number range for the current solar pump to higher values encroaching into the inertially-dominant range. Finally, supplementary electromagnetic phenomena may also be examined in refinements of the present solar magnetic nanofluid peristaltic pump model, including Hall currents, Maxwell displacement currents, magnetic induction, Joule heating and ion slip. 
APPENDIX 1: ANALYTICAL SOLUTIONS

$$
\begin{aligned}
& c_{1}=\left(\frac{N b+N t}{N b\left(h_{1}-h_{2}\right)}\right), c_{2}=-c_{1} h_{2}, \xi=\left(\frac{1+R n \operatorname{Pr}}{\operatorname{Pr}}\right), A_{1}=\frac{c_{1} N b}{\xi}, A_{2}=\frac{\beta}{\xi}, A_{3}=\frac{A_{1}+A_{2}\left(h_{1}-h_{2}\right)}{A_{1}\left(e^{-A_{1} h_{2}}-e^{-A_{1} h_{1}}\right)}, \\
& A_{4}=\frac{\left(A_{1} e^{-A_{1} h_{2}}+A_{2} h_{1} e^{-A_{1} h_{2}}-A_{2} h_{2} e^{-A_{1} h_{1}}\right)}{A_{1}\left(e^{-A_{1} h_{1}}-e^{-A_{1} h_{2}}\right)}, A_{5}=\frac{\left(N b+A_{3} N t\left(e^{-A_{1} h_{1}}-e^{-A_{1} h_{2}}\right)\right)}{N b\left(h_{1}-h_{2}\right)}, A_{6}=\frac{A_{3} N t}{N b}, \\
& A_{7}=\frac{\left(N b h_{2}-A_{3} N t h_{1} e^{-A_{1} h_{2}}+A_{3} N t h_{2} e^{-A_{1} h_{1}}\right)}{N b\left(h_{1}-h_{2}\right)}, A_{8}=\frac{G r A_{2}}{A_{1}-B r A_{5}}, A_{9}=-\frac{B r A_{1} A_{6}}{G r A_{1} A_{3}} \\
& L_{10}=-\frac{A_{8}}{2 N^{2}}, L_{11}=\left(A_{1}^{4}-N^{2} A_{1}^{2}\right), \\
& D_{1}=N^{3} N b^{2} c_{1} \xi^{5}\left(h_{1}-h_{2}\right)\left(4 \cosh \left(N\left(h_{1}-h_{2}\right)\right)-2 N h_{1} \sinh \left(N\left(h_{1}-h_{2}\right)\right)+2 N h_{2} \sinh \left(N\left(h_{1}-h_{2}\right)\right)-4\right) \text {, } \\
& D_{2}=4 N^{3} N b^{2} c_{1} \xi^{5}\left(2 \cosh \left(N\left(h_{1}-h_{2}\right)\right)-N h_{1} \sinh \left(N\left(h_{1}-h_{2}\right)\right)+N h_{2} \sinh \left(N\left(h_{1}-h_{2}\right)\right)-2\right), \\
& D_{3}=2 N^{2} N b^{7} c_{1}^{4}\left(h_{1}-h_{2}\right)+2 N^{3} N b^{6} c_{1}^{5}\left(h_{1}-h_{2}\right)-2 N^{4} N b^{5} c_{1}^{4}\left(\xi^{2}-\xi^{3}\right)\left(h_{1}-h_{2}\right), \\
& D_{4}=8 N^{2} N b^{2} c_{1} \xi^{5}\left(h_{1}-h_{2}\right)+4 B r N t \beta \xi^{5}\left(h_{1}^{2}-h_{2}^{2}\right)+4 B r N b^{2} c_{1} \xi^{5}\left(h_{1}+h_{2}\right)-4 G r N b \beta \xi^{5}\left(h_{1}^{2}-h_{2}^{2}\right) \\
& D_{5}=2 N b^{2} c_{1} \xi^{5}\left(h_{1}-h_{2}\right)\left(4 \cosh \left(N\left(h_{1}-h_{2}\right)\right)-2 N h_{1} \sinh \left(N\left(h_{1}-h_{2}\right)\right)+2 N h_{2} \sinh \left(N\left(h_{1}-h_{2}\right)\right)-4\right) \text {, } \\
& c_{5}=-\left(2 N^{3} N b^{2} c_{1}\left(h_{1}^{2}-h_{2}^{2}\right)+B r N N b N t c_{1}\left(h_{1}^{2}-h_{2}^{2}\right)+B r N N t \beta h_{1} h_{2}\left(h_{1}-h_{2}\right)-G r N N b \beta h_{1} h_{2}\left(h_{1}-h_{2}\right)\right. \\
& -\left(2 B r N t \beta\left(h_{1}^{2}+h_{2}^{2}\right)-2 B r N b c_{1}\left(h_{1}^{2}-h_{2}^{2}\right)+2 G r N b \beta\left(h_{1}^{2}+h_{2}^{2}\right)-2 B r N b N t c_{1}\left(h_{1}-h_{2}\right)\right. \\
& +4(B r N t-G r N b) \beta h_{1} h_{2}-q_{0} N^{4} N b^{2} c_{1}\left(h_{1}^{2}-h_{2}^{2}\right)+B r N^{2} N t \beta\left(h_{1}^{3}+h_{2}^{3}\right)-G r N^{2} N b \beta h_{1} h_{2}\left(h_{1}^{2}+h_{2}^{2}\right) \\
& -2 B r N^{2} N t \beta h_{1}^{2} h_{2}^{2}-B r N^{2} N b^{2} c_{1} h_{1} h_{2}\left(h_{1}-h_{2}\right)+2 G r N^{2} N b \beta h_{1}^{2} h_{2}^{2} \\
& \left.-\operatorname{BrN}^{2} N b N t c_{1} h_{1} h_{2}\left(h_{1}-h_{2}\right)\right) \sinh \left(N\left(h_{1}-h_{2}\right)\right)+\operatorname{BrNNt} \beta\left(h_{1}^{3}-h_{2}^{3}\right)-\operatorname{GrNNb} \beta\left(h_{1}^{3}-h_{2}^{3}\right) \\
& +\operatorname{BrNNbc}_{1}\left(h_{1}^{2}-h_{2}^{2}\right)+\left(\operatorname{BrNNt} \beta\left(h_{1}^{3}-h_{2}^{3}\right)-2 N^{3} N b^{2} c_{1}\left(h_{1}^{2}-h_{2}^{2}\right)-G r N N b \beta\left(h_{1}^{3}-h_{2}^{3}\right)\right. \\
& +\operatorname{BrNNb}^{2} c_{1}\left(h_{1}^{2}-h_{2}^{2}\right)+\operatorname{BrNNbN}_{t} c_{1}\left(h_{1}^{2}-h_{2}^{2}\right)+5 B r N N t \beta h_{1} h_{2}\left(h_{1}-h_{2}\right)-4 B r M N b^{2} c_{1} h_{1} h_{2} \\
& \left.\left.-5 G r N N b \beta h_{1} h_{2}\left(h_{1}-h_{2}\right)-4 B r N N b N t c_{1} h_{1} h_{2}\right) \cosh \left(N\left(h_{1}-h_{2}\right)\right)\right) / D_{1}
\end{aligned}
$$




$$
\begin{aligned}
& -\xi^{2}\left(\left(2 N^{5} N b^{5} c_{1}^{4}\left(h_{1}^{2}-h_{2}^{2}\right)-2 N^{5} N b^{5} c_{1}^{4} h_{1} h_{2}\right)\left(e^{-A_{1} h_{1}}-e^{-A_{1} h_{2}}\right)\right. \\
& +\left(N^{5} N b^{5} c_{1}^{4} h_{2}\left(h_{2}-h_{1}\right)-N^{5} N b^{5} c_{1}^{4} h_{2}\left(h_{2}-h_{1}\right)\right)\left(e^{N\left(h_{1}-h_{2}\right)-A_{1} h_{1}}-e^{-N\left(h_{1}-h_{2}\right)-A_{1} h_{1}}\right) \\
& +\left(N^{5} N b^{5} c_{1}^{4} h_{1}\left(h_{2}-h_{1}\right)-N^{5} N b^{5} c_{1}^{4} h_{2}\left(h_{2}-h_{1}\right)\right)\left(e^{N\left(h_{1}-h_{2}\right)-A_{1} h_{2}}-e^{-N\left(h_{1}-h_{2}\right)-A_{1} h_{2}}\right) \\
& \left.-\left(2 N^{3} N b^{7} c_{1}^{6}\left(h_{1}^{2}-h_{2}^{2}\right)-2 N^{3} N b^{7} c_{1}^{6} h_{1} h_{2}\right)\left(e^{-A_{1} h_{1}}-e^{-A_{1} h_{2}}\right)\right) / D_{1} \\
& -\xi\left(\left(2 N^{3} N b^{3} c_{1}^{5}\left(h_{1}-h_{2}\right)\right)\left(e^{-A_{1} h_{1}}-e^{-A_{1} h_{2}}\right)\right. \\
& +\left(N^{4} N b^{6} c_{1}^{5} h_{2}\left(h_{2}-h_{1}\right)+N^{3} N b^{2} c_{1}^{5}\left(h_{2}-h_{1}\right)+N^{3} N b^{7} c_{1}^{6} h_{2}\left(h_{2}-h_{1}\right)+N^{2} N b^{7} c_{1}^{6}\left(h_{2}-h_{1}\right)\right) \\
& \left(e^{N\left(h_{1}-h_{2}\right)-A_{1} h_{1}}-e^{-N\left(h_{1}-h_{2}\right)-A_{1} h_{1}}\right) \\
& +\left(N^{4} N b^{5} c_{1}^{5} h_{1}\left(h_{1}-h_{2}\right)-N^{3} N b^{6} c_{1}^{5}\left(h_{1}-h_{2}\right)+N^{3} N b^{7} c_{1}^{6} h_{1}\left(h_{1}-h_{2}\right)-N^{2} N b^{7} c_{1}^{6}\left(h_{1}-h_{2}\right)\right) \\
& \left.\left(e^{N\left(h_{1}-h_{2}\right)-A_{1} h_{2}}-e^{-N\left(h_{1}-h_{2}\right)-A_{1} h_{2}}\right)\right) / D_{1}+\xi^{3}\left(\left(2 N^{5} N b^{4} c_{1}^{3}\left(h_{1}-h_{2}\right)\right)\left(e^{-A_{1} h_{1}}-e^{-A_{1} h_{2}}\right)\right. \\
& +\left(N^{6} N b^{4} c_{1}^{3} h_{2}\left(h_{2}-h_{1}\right)+N^{5} N b^{4} c_{1}^{3}\left(h_{2}-h_{1}\right)\right)\left(e^{N\left(h_{1}-h_{2}\right)-A_{1} h_{1}}-e^{-N\left(h_{1}-h_{2}\right)-A_{1} h_{1}}\right) \\
& \left.+\left(N^{6} N b^{4} c_{1}^{3} h_{1}\left(h_{1}-h_{2}\right)-N^{5} N b^{4} c_{1}^{3}\left(h_{1}-h_{2}\right)\right)\left(e^{N\left(h_{1}-h_{2}\right)-A_{1} h_{2}}-e^{-N\left(h_{1}-h_{2}\right)-A_{1} h_{2}}\right)\right) / D_{1}, \\
& c_{6}=\left(2 N^{4} N b^{5} c_{1}^{4} \xi^{2}+2 N^{5} N b^{4} c_{1}^{3} \xi^{3}-2 N^{3} N b^{6} c_{1}^{5} \xi-2 N^{2} N b^{7} c_{1}^{6}\right)\left(e^{N h_{1}-A_{1} h_{1}}+e^{N h_{2}-A_{1} h_{2}}\right) \\
& +\left(2 N^{2} N b^{7} c_{1}^{6}+2 N^{3} N b^{7} c_{1}^{6}\left(h_{2}-h_{1}\right)+2 N^{3} N b^{6} c_{1}^{5} \xi+2 N^{4} N b^{5} c_{1}^{4} \xi^{2}-2 N^{4} N b^{4} c_{1}^{3} \xi^{3}\right. \\
& \left.+2 N^{5} N b^{5} c_{1}^{4} \xi^{2}\left(h_{1}-h_{2}\right)\right)\left(e^{N h_{1}-A_{1} h_{2}}+e^{N h_{2}-A_{1} h_{1}}\right)+2 B r N N t \beta h_{1} h_{2} \xi^{5} \\
& +\left(2 B r N b^{2} c_{1} \xi^{5}+2 B r N t \beta\left(h_{1}-h_{2}\right) \xi^{5}-2 G r N b \beta\left(h_{1}-h_{2}\right) \xi^{5}+2 q_{0} M^{3} N b^{2} c_{1} \xi^{2}\right. \\
& \left.+2 N^{3} N b^{2} c_{1} \xi^{5}\left(h_{1}-h_{2}\right)+2 B r N b N t c_{1} \xi^{5}\right)\left(e^{N h_{1}}-e^{N h_{2}}\right) \\
& +\left(B r N N b^{2} c_{1}\left(h_{2}-h_{1}\right) \xi^{5}+G r N N b \beta \xi^{5}\left(h_{1}^{2}-h_{2}^{2}\right)-\operatorname{BrNNbNt}_{1} \beta \xi^{5}\left(h_{1}-h_{2}\right)\right. \\
& \left.\left.-2 G r N N b \beta h_{1} h_{2} \xi^{5}-\operatorname{BrNNt} \beta \xi^{5}\left(h_{1}^{2}-h_{2}^{2}\right)\right)\left(e^{N h_{1}}+e^{N h_{2}}\right)\right) / D_{2} \text {, } \\
& c_{7}=\left(2 N^{4} N b^{5} c_{1}^{4} \xi^{2}+2 N^{5} N b^{4} c_{1}^{3} \xi^{3}-2 N^{3} N b^{6} c_{1}^{5} \xi-2 N^{2} N b^{7} c_{1}^{6}\right)\left(e^{-N h_{1}-A_{1} h_{1}}+e^{-N h_{2}-A_{1} h_{2}}\right) \\
& +\left(2 N^{2} N b^{7} c_{1}^{6}+2 N^{3} N b^{7} c_{1}^{6}\left(h_{2}-h_{1}\right)+2 N^{3} N b^{6} c_{1}^{5} \xi+2 N^{4} N b^{5} c_{1}^{4} \xi^{2}-2 N^{4} N b^{4} c_{1}^{3} \xi^{3}\right. \\
& \left.+2 N^{5} N b^{5} c_{1}^{4} \xi^{2}\left(h_{1}-h_{2}\right)\right)\left(e^{-N h_{1}-A_{1} h_{2}}+e^{-N h_{2}-A_{1} h_{1}}\right) \\
& +\left(2 B r N b^{2} c_{1} \xi^{5}+2 B r N t \beta\left(h_{1}-h_{2}\right) \xi^{5}-2 G r N b \beta\left(h_{1}-h_{2}\right) \xi^{5}+2 q_{0} N^{3} N b^{2} c_{1} \xi^{2}\right. \\
& \left.+2 N^{3} N b^{2} c_{1} \xi^{5}\left(h_{1}-h_{2}\right)+2 B r N b N t c_{1} \xi^{5}\right)\left(e^{-N h_{1}}-e^{-N h_{2}}\right)+\left(B r N N b^{2} c_{1}\left(h_{2}-h_{1}\right) \xi^{5}\right. \\
& +\operatorname{GrNN} N \beta \xi^{5}\left(h_{1}^{2}-h_{2}^{2}\right)-B r N N b N t c_{1} \beta \xi^{5}\left(h_{1}-h_{2}\right)+2 B r N N t \beta h_{1} h_{2} \xi^{5} \\
& \left.\left.-2 G r M N b \beta h_{1} h_{2} \xi^{5}-B r M N_{t} \beta \xi^{5}\left(h_{1}^{2}-h_{2}^{2}\right)\right)\left(e^{-M h_{1}}+e^{-M h_{2}}\right)\right) / D_{2} \text {, }
\end{aligned}
$$




$$
\begin{aligned}
& c_{8}=\left(D_{3}\left(e^{N h_{1}-N h_{2}-A_{1} h_{1}}+e^{-N h_{1}+N h_{2}-A_{1} h_{1}}+e^{N h_{1}-N h_{2}-A_{1} h_{2}}+e^{-N h_{1}+N h_{2}-A_{1} h_{2}}\right)\right. \\
& -\left(4 N^{2} N b^{7} c_{1}^{6}\left(h_{1}-h_{2}\right)-2 N^{4} N b^{5} c_{1}^{4} \xi^{2}\left(h_{1}-h_{2}\right)\right)\left(e^{-A_{1} h_{1}}+e^{-A_{1} h_{2}}\right) \\
& +D_{4}-D_{4}\left(\cosh \left(N\left(h_{1}-h_{2}\right)\right)\right) \\
& +\left(2 B r N N t \beta \xi^{5}\left(h_{1}^{3}+h_{2}^{3}\right)-2 G r N N b \beta \xi^{5}\left(h_{1}^{3}+h_{2}^{3}\right)+2 B r N N b^{2} c_{1} \beta \xi^{5}\left(h_{1}^{2}-h_{2}^{2}\right)\right. \\
& -4 q_{0} N^{3} N b^{2} c_{1} \xi^{5}\left(h_{1}-h_{2}\right)-4 B r N b N t c_{1} \xi^{5}\left(h_{1}+h_{2}\right)+2 B r N N b N t c_{1} \xi^{5}\left(h_{1}^{2}-h_{2}^{2}\right) \\
& \left.-2 B r N N t N b \beta h_{1} h_{2} \xi^{5}\left(h_{1}+h_{2}\right)+2 G r N N b \beta h_{1} h_{2} \xi^{5}\left(h_{1}+h_{2}\right)\right) \sinh \left(N\left(\left(h_{1}-h_{2}\right)\right)\right) / D_{5} \text {, } \\
& B_{1}=2 A_{1}^{2} A_{2} L_{11}-A_{1}^{2} A_{2} L_{11}-A_{1}^{4} A_{4} L_{11}-2 A_{1} A_{3} L_{10}, B_{2}=-\frac{2 A_{2} L_{10}}{A_{1}}, B_{3}=3 A_{1}^{4} A_{3} L_{11}-A_{1}^{3} A_{3} L_{11} \\
& B_{4}=-A_{1}^{3} A_{2} L_{11}, B_{5}=\frac{2 A_{2} c_{6} N^{3}}{A_{1}}-\frac{A_{2} c_{6} N^{2}}{A_{1}}-\frac{A_{2} c_{6} N^{4}}{A_{1}}, B_{6}=-\frac{2 A_{2} c_{7} N^{3}}{A_{1}}-\frac{A_{2} c_{7} N^{2}}{A_{1}}-A_{4} c_{6} N^{4}, \\
& B_{7}=-\frac{A_{2} c_{7} N^{4}}{A_{1}}, B_{8}=-A_{1} A_{3} c_{6} N^{2}+2 A_{1} A_{3} c_{6} N^{3}+A_{3} c_{6} N^{4}, B_{9}=-A_{1} A_{3} c_{7} N^{2}-2 A_{1} A_{3} c_{7} N^{3}+A_{3} c_{6} N^{4} \\
& Z_{1}=A_{1}^{4}-N^{2} A_{1}^{2}, Z_{2}=A_{1}^{3}, Z_{3}=A_{1}^{2}-N^{2}, Z_{4}=Z_{2} Z_{3}^{2}, Z_{5}=4 B_{4}\left(A_{1}^{2}-2 N^{2}\right), \\
& L_{1}=\frac{B_{1}}{Z_{1}}+\frac{Z_{5}}{Z_{4}}, L_{2}=-\frac{B_{2}}{2 N^{2}}, Z_{6}=16 A_{1}^{4}-4 N^{2} A_{1}^{2}, L_{3}=\frac{B_{3}}{Z_{6}}, L_{4}=\frac{B_{4}}{Z_{1}}, L_{5}=-\frac{B_{5}}{2 N^{3}}, \\
& L_{6}=\frac{B_{6}}{2 N^{3}}+\frac{5 B_{7}}{N^{4}}, L_{7}=-\frac{B_{7}}{4 N^{3}}, Z_{7}=\left(A_{1}+N\right)^{4}, Z_{8}=\left(A_{1}+N\right)^{2}, Z_{9}=Z_{8} N^{2}, \\
& Z_{10}=Z_{7}-Z_{9}, \quad L_{8}=\frac{B_{8}}{Z_{10}}, Z_{11}=\left(A_{1}-N\right)^{4}, Z_{12}=\left(A_{1}-N\right)^{2}, Z_{13}=Z_{12} N^{2}, \\
& Z_{14}=Z_{11}-Z_{13}, \quad L_{9}=\frac{B_{9}}{Z_{14}} \\
& D_{6}=N \xi\left(4 \cosh \left(N h_{1}-N h_{2}\right)-2 N\left(h_{1}-h_{2}\right) \sinh \left(N h_{1}-N h_{2}\right)-4\right) \text {, } \\
& D_{7}=2 \xi\left(2 \cosh \left(M h_{1}-M h_{2}\right)-M\left(h_{1}-h_{2}\right) \sinh \left(M h_{1}-M h_{2}\right)-2\right),
\end{aligned}
$$




$$
\begin{aligned}
& c_{9}=\left(2 L_{9} N b c_{1}-4 L_{9} N \xi+4 L_{9} N^{2} h_{1} \xi-2 L_{9} N N b h_{1} c_{1}\right)\left(e^{N h_{1}-A_{1} h_{2}}+e^{N h_{2}-A_{1} h_{1}}\right) \\
& -\left(2 L_{8} N b c_{1}+4 L_{8} N \xi+4 L_{8} N^{2} h_{2} \xi+2 L_{8} N N b h_{2} c_{1}\right)\left(e^{N h_{2}-A_{1} h_{1}}+e^{-N h_{1}-A_{1} h_{2}}\right) \\
& +\left(2 L_{3} N b c_{1}-2 L_{3} N \xi+2 L_{3} N^{2} h_{1} \xi-4 L_{3} N N b h_{1} c_{1}\right)\left(e^{N h_{1}-N h_{2}-2 A_{1} h_{2}}\right) \\
& -\left(4 L_{3} N b c_{1}+2 L_{3} N \xi+2 L_{3} N^{2} h_{1} \xi+4 L_{3} N N b h_{1} c_{1}\right)\left(e^{N h_{1}-N h_{2}-2 A_{1} h_{1}}+e^{N h_{2}-N h_{1}-2 A_{1} h_{2}}\right) \\
& +\left(4 L_{3} N b c_{1}-2 L_{3} N \xi+2 L_{3} N^{2} h_{2} \xi+4 L_{3} N N b h_{2} c_{1}\right)\left(e^{N h_{2}-N h_{1}-2 A_{1} h_{1}}\right) \\
& -\left(4 L_{7} \xi N^{2} h_{1}^{2}-4 L_{7} h_{2} N^{2} \xi-2 L_{7} N h_{1} \xi+4 L_{7} N h_{2} \xi+2 L_{7} \xi\right) e^{N h_{1}} \\
& -\left(4 L_{7} \xi N^{2} h_{2}^{2}-4 L_{7} h_{1} N^{2} \xi-2 L_{7} N h_{2} \xi+4 L_{7} N h_{1} \xi+2 L_{7} \xi\right) e^{N h_{2}} \\
& +\left(4 L_{4} N N b c_{1} h_{1}^{2}+4 L_{1} h_{1} N N b c_{1}+4 L_{1} N \xi\right) e^{-A_{1} h_{1}} \\
& +\left(4 L_{4} N N b c_{1} h_{2}^{2}+4 L_{1} h_{2} N N b c_{1}+4 L_{1} N \xi\right) e^{-A_{1} h_{2}} \\
& +\left(2 L_{8} N b c_{1}-2 L_{8} N N b h_{2} c_{1}\right)\left(e^{N h_{2}-2 N h_{1}-A_{1} h_{1}}+e^{N h_{1}-2 N h_{2}-A_{1} h_{2}}\right) \\
& -\left(2 L_{9} N b c_{1}+2 L_{9} N N b h_{2} c_{1}\right)\left(e^{2 N h_{1}-M h_{2}-A_{1} h_{1}}+e^{2 N h_{2}-N h_{1}-A_{1} h_{2}}\right) \\
& +\left(4 L_{8} N \xi+4 L_{8} N^{2} h_{1} \xi+4 L_{8} N N b c_{1} h_{1}\right)\left(e^{-N h_{1}-A_{1} h_{1}}\right) \\
& +\left(4 L_{9} N \xi-4 L_{9} N^{2} h_{1} \xi+4 L_{9} N N b c_{1} h_{1}\right)\left(e^{N h_{1}-A_{1} h_{1}}\right) \\
& +\left(4 L_{8} N \xi+4 L_{8} N^{2} h_{2} \xi+4 L_{8} N N b c_{1} h_{2}\right)\left(e^{-N h_{2}-A_{1} h_{2}}\right) \\
& +\left(4 L_{9} N \xi-4 L_{9} N^{2} h_{2} \xi+4 L_{9} N N b c_{1} h_{2}\right)\left(e^{N h_{2}-A_{1} h_{2}}\right) \\
& +\left(2 L_{7} \xi+2 L_{7} N h_{1}\right)\left(e^{2 N h_{2}-N h_{1}}\right)+\left(2 L_{7} \xi+2 L_{7} N h_{2}\right)\left(e^{2 N h_{1}-N h_{2}}\right) \\
& -\left(\begin{array}{l}
2 L_{1} N b c_{1}-2 L_{4} N \xi+2 L_{1} N^{2} h_{2} \xi+2 L_{4} N b h_{1} c_{1}-2 L_{4} N h_{1} \xi \\
-2 L_{4} N h_{2} \xi+2 L_{1} N N b h_{2} c_{1}+2 L_{4} N^{2} h_{1} h_{2} \xi+2 L_{4} N N b h_{1} h_{2} \xi
\end{array}\right)\left(e^{N h_{1}-N h_{2}-A_{1} h_{1}}\right) \\
& -\left(\begin{array}{l}
2 L_{4} \xi-2 L_{1} N b c_{1}+2 L_{1} N \xi-2 L_{1} N^{2} h_{2} \xi \\
+2 L_{4} N b c_{1} h_{1}+2 L_{4} N h_{1} \xi-2 L_{4} N h_{2} \xi+2 L_{1} N N b c_{1} h_{2} \\
-2 L_{4} N^{2} h_{1} h_{2} \xi+2 L_{4} N N b c_{1} h_{1} h_{2}
\end{array}\right)\left(e^{N h_{2}-N h_{1}-A_{1} h_{1}}\right) \\
& -\left(\begin{array}{l}
2 L_{4} \xi-2 L_{1} N b c_{1}+2 L_{1} M \xi-2 L_{1} N^{2} h_{1} \xi \\
-2 L_{4} N b c_{1} h_{1}-2 L_{4} N h_{1} \xi+2 L_{4} N h_{2} \xi+2 L_{1} N N b c_{1} h_{1} \\
-2 L_{4} N^{2} h_{1} h_{2} \xi+2 L_{4} N N b c_{1} h_{1} h_{2}
\end{array}\right)\left(e^{N h_{1}-N h_{2}-A_{1} h_{2}}\right) \\
& -\left(\begin{array}{l}
2 L_{1} N b c_{1}-2 L_{4} \xi+2 L_{1} N \xi+2 L_{1} N^{2} h_{1} \xi+2 L_{4} N b h_{2} c_{1} \\
-2 L_{4} N h_{1} \xi+2 L_{4} N h_{2} \xi+2 L_{1} N N b c_{1} h_{1}+2 L_{4} N^{2} h_{1} h_{2} \xi+2 L_{4} N N b c_{1} h_{1} h_{2}
\end{array}\right)\left(e^{N h_{2}-N h_{1}-A_{1} h_{1}}\right) \\
& +\left(4 L_{3} N \xi+8 L_{3} N N b c_{1} h_{1}\right) e^{-2 A_{1} h_{1}}+\left(4 L_{3} N \xi+8 L_{3} N N b c_{1} h_{2}\right) e^{-2 A_{1} h_{2}} \\
& +8 L_{2} N \xi\left(1-\cosh \left(N h_{1}-N h_{2}\right)\right)+2 q_{1} N^{2}\left(h_{1}-h_{2}\right) \xi+4 L_{2} N^{2} h_{1} \xi \sinh \left(N h_{1}-N h_{2}\right),
\end{aligned}
$$


44

$$
\begin{aligned}
& c_{10}=-\left(\left(L_{8} N b c_{1}+2 L_{8} N \xi+L_{8} N^{2} h_{1} \xi-L_{8} N^{2} h_{2} \xi+L_{8} N N b\left(h_{1}-h_{2}\right) c_{1}\right)\left(e^{N h_{2}-N h_{1}-A_{1} h_{1}}\right)\right. \\
& -\left(L_{8} N b c_{1}+2 L_{8} N \xi\right)\left(e^{-A_{1} h_{2}}+e^{-A_{1} h_{1}}\right) \\
& +\left(L_{8} N b c_{1}+2 L_{8} N \xi+L_{8} N^{2} h_{1} \xi-L_{8} N^{2} h_{2} \xi+L_{8} N N b\left(h_{1}-h_{2}\right) c_{1}\right)\left(e^{N h_{2}-N h_{1}-A_{1} h_{1}}+e^{N h_{1}-N h_{2}-A_{1} h_{2}}\right) \\
& -\left(L_{1} N b c_{1}-L_{4} \xi+L_{1} N \xi+L_{4} N b c_{1} h_{1}+L_{4} N h_{1} \xi\right)\left(e^{N h_{1}-A_{1} h_{1}}\right) \\
& -\left(L_{1} N b c_{1}-L_{4} \xi+L_{1} N \xi+L_{4} N b c_{1} h_{2}+L_{4} N h_{2} \xi\right)\left(e^{N h_{2}-A_{1} h_{2}}\right) \\
& +\left(\begin{array}{c}
L_{1} N b c_{1}-L_{4} \xi+L_{1} N \xi+L_{4} N b c_{1} h_{1}+L_{4} N h_{2} \xi \\
+L_{1} N N b c_{1}\left(h_{1}-h_{2}\right)+L_{4} N N b c_{1} h_{1}\left(h_{1}-h_{2}\right)
\end{array}\right)\left(e^{N h_{2}-A_{1} h_{1}}\right) \\
& +\left(\begin{array}{c}
L_{1} N b c_{1}-L_{4} \xi+L_{1} N \xi+L_{4} N b c_{1} h_{2}+L_{4} N h_{1} \xi \\
-L_{1} N N b c_{1}\left(h_{1}-h_{2}\right)+L_{4} N N b c_{1} h_{2}\left(h_{1}-h_{2}\right)
\end{array}\right)\left(e^{N h_{1}-A_{1} h_{2}}\right) \\
& -\left(L_{7} \xi N^{2} h_{1}^{2}+h_{2}^{2}-2 L_{7} h_{1} h_{2} N^{2} \xi+2 L_{7} \xi\right)\left(e^{N h_{1}}+e^{N h_{2}}\right) \\
& -\left(2 L_{3} N b c_{1}+L_{3} N \xi\right)\left(e^{N h_{1}-2 A_{1} h_{1}}+e^{N h_{2}-2 A_{1} h_{2}}\right) \\
& +\left(2 L_{3} N b c_{1}+L_{3} N \xi+2 L_{3} N N b c_{1} h_{1}-2 L_{3} N N b c_{1} h_{2}\right)\left(e^{N h_{1}-2 A_{1} h_{2}}+e^{N h_{2}-2 A_{1} h_{1}}\right) \\
& +\left(L_{9} N b c_{1}-L_{9} N^{2} h_{1} \xi+L_{9} N^{2} h_{2} \xi+L_{9} N N b c_{1}\left(h_{1}-h_{2}\right)\right)\left(e^{N h_{1}+N h_{2}-A_{1} h_{1}}\right) \\
& +\left(L_{9} N b c_{1}+L_{9} N^{2} h_{1} \xi-L_{9} N^{2} h_{2} \xi-L_{9} N N b c_{1}\left(h_{1}-h_{2}\right)\right)\left(e^{N h_{1}+N h_{2}-A_{1} h_{2}}\right) \\
& +\left(L_{7} \xi\right)\left(e^{2 N h_{1}}+e^{2 N h_{2}}\right)+4 L_{5} N \xi \cosh \left(N h_{1}-N h_{2}\right) \\
& -L_{9} N b c_{1}\left(e^{N h_{1}-A_{1} h_{1}}+e^{2 N h_{2}-A_{1} h_{2}}\right)+q_{1} N \xi\left(e^{N h_{1}}-e^{N h_{2}}\right) \\
& \left.-2 L_{5} N^{2}\left(h_{1}-h_{2}\right) \xi \sinh \left(N\left(h_{1}-h_{2}\right)\right)-4 L_{5} N \xi\right) / D_{6} \text {, }
\end{aligned}
$$




$$
\begin{aligned}
& c_{11}=\left(2 L_{9} N-L_{9} N^{2}\left(h_{1}-h_{2}\right)\right)\left(e^{N h_{1}-N h_{2}-A_{1} h_{1}}-e^{N h_{2}-N h_{1}-A_{1} h_{2}}\right) \\
& -L_{8} N^{2}\left(h_{1}-h_{2}\right)\left(e^{-N h_{1}-N h_{2}-A_{1} h_{1}}-e^{-N h_{1}-N h_{2}-A_{1} h_{2}}\right)+\left(L_{4}+L_{1} N+L_{4} N h_{1}+L_{1} N_{b} c_{1}\right)\left(e^{-N h_{1}-A_{1} h_{1}}-e^{-N h_{1}-A_{1} h_{2}}\right) \\
& -\left(L_{4}+L_{1} N+L_{4} N h_{2}-L_{1} N b c_{1}-2 L_{3} N b c_{1}\right)\left(e^{-N h_{2}-A_{1} h_{1}}-e^{-N h_{2}-A_{1} h_{2}}\right)-2 L_{7} \cosh \left(N\left(h_{1}-h_{2}\right)\right)+4 L_{6} N+2 L_{7} \\
& +\left(L_{3} N+2 L_{3} N b c_{1}\right)\left(e^{-N h_{1}-2 A_{1} h_{1}}-e^{-N h_{1}-2 A_{1} h_{2}}\right)+2 L_{7} N\left(h_{1}+h_{2}\right)-q_{1} N\left(e^{-N h_{1}}-e^{-N h_{2}}\right) \\
& +\left(2 L_{9} N-2 L_{9} N b c_{1}\right)\left(e^{-A_{1} h_{1}}+e^{-A_{1} h_{2}}\right)-4 L_{6} N \cosh \left(N h_{1}-N h_{2}\right) \\
& +\left(L_{7} N^{2}\left(h_{1}^{2}-h_{1}^{2}\right)\right)\left(e^{N h_{1}-N h_{2}}-e^{N h_{2}-N h_{1}}\right)-2 L_{7} N\left(h_{1}+h_{2}\right) \cosh \left(N h_{1}-N h_{2}\right) \\
& +2 L_{6} N^{2}\left(h_{1}-h_{2}\right) \sinh \left(N h_{1}-N h_{2}\right)-2 L_{7} N^{2} h_{1} h_{2} \cosh \left(N h_{1}-N h_{2}\right) \\
& +\left(L_{8} N b c_{1}\right)\left(e^{-2 N h_{1}-A_{1} h_{1}}+e^{-2 N h_{2}-2 A_{1} h_{2}}\right)-\left(L_{8} N b c_{1}\right)\left(e^{-N h_{1}-N h_{2}-A_{1} h_{1}}\right)-L_{9} N b c_{1}\left(e^{M h_{1}-M h_{2}-A_{1} h_{1}}\right) \\
& +\left(L_{4} N b c_{1}\left(h_{1}-h_{2}\right)\right)\left(e^{-N h_{1}-A_{1} h_{1}}-e^{-N h_{2}-A_{1} h_{1}}\right) \\
& -\left(L_{1} N N b c_{1}\left(h_{1}-h_{2}\right)\right)\left(e^{-N h_{1}-A_{1} h_{2}}-e^{-N h_{2}-A_{1} h_{1}}\right)-\left(2 L_{3} N N b c_{1}\left(h_{1}-h_{2}\right)\right)\left(e^{-N h_{1}-2 A_{1} h_{2}}-e^{-N h_{2}-2 A_{1} h_{1}}\right) \\
& +\left(L_{8} N N b c_{1}\left(h_{1}-h_{2}\right)\right)\left(e^{-N h_{1}-M h_{2}-2 A_{1} h_{1}}-e^{-N h_{2}-2 A_{1} h_{2}}\right) \\
& +\left(L_{9} N N b c_{1}\left(h_{1}-h_{2}\right)\right)\left(e^{N h_{1}-N h_{2}-2 A_{1} h_{1}}-e^{N h_{2}-2 A_{1} h_{2}}\right) \\
& +\left(L_{4} N N b c_{1} h_{1}^{2}-h_{1} h_{2}\right)\left(e^{-N h_{2}-A_{1} h_{1}}\right)+\left(L_{4} M N_{b} c_{1} h_{2}^{2}-h_{1} h_{2}\right)\left(e^{-M h_{1}-A_{1} h_{2}}\right) / D_{6}, \\
& c_{12}=-\left(\left(L_{7} \xi+2 L_{7} N\left(h_{1}-h_{2}\right) \xi\right) N^{2} e^{N h_{1}}+\left(L_{7} \xi-2 L_{7} N\left(h_{1}+h_{2}\right) \xi\right) e^{N h_{2}}\right. \\
& +\left(L_{1} N b c_{1}-L_{4} \xi+L_{1} N \xi+L_{4} N b c_{1} h_{1}+L_{4} N h_{1} \xi\right) e^{N h_{1}-N h_{2}-A_{1} h_{1}} \\
& -\left(L_{4} \xi-L_{1} N b c_{1}+L_{1} N \xi-L_{4} N b c_{1} h_{1}+L_{4} N h_{1} \xi\right) e^{N h_{2}-N h_{1}-A_{1} h_{1}} \\
& +\left(L_{1} N b c_{1}-L_{4} \xi+L_{1} N \xi+L_{4} N b c_{1} h_{2}+L_{4} N h_{2} \xi\right) e^{N h_{2}-N h_{1}-A_{1} h_{2}} \\
& -\left(L_{4} \xi-L_{1} N b c_{1}+L_{1} N \xi-L_{4} N b c_{1} h_{2}+L_{4} N h_{2} \xi\right) e^{N h_{1}-N h_{2}-A_{1} h_{2}} \\
& +\left(2 L_{8} N b c_{1}+2 L_{8} N \xi\right)\left(e^{-N h_{1}-A_{1} h_{2}}+e^{-N h_{2}-A_{1} h_{1}}\right)+\left(2 L_{9} N b c_{1}-2 L_{9} N \xi\right)\left(e^{N h_{1}-A_{1} h_{2}}+e^{N h_{2}-A_{1} h_{1}}\right) \\
& -\left(2 L_{8} N b c_{1}+2 L_{8} N \xi\right)\left(e^{-N h_{1}-A_{1} h_{1}}+e^{-N h_{2}-A_{1} h_{2}}\right)-\left(2 L_{9} N b c_{1}-2 L_{9} N \xi\right)\left(e^{N h_{1}-A_{1} h_{1}}+e^{N h_{2}-A_{1} h_{2}}\right) \\
& -\left(2 L_{1} N b c_{1}-2 L_{4} \xi+2 L_{4} N b c_{1} h_{1}\right) e^{-A_{1} h_{1}}-\left(2 L_{1} N b c_{1}-2 L_{4} \xi+2 L_{4} N b c_{1} h_{2}\right) e^{-A_{1} h_{2}} \\
& +\left(2 L_{3} N b c_{1}+L_{3} N \xi\right)\left(e^{N h_{1}-N h_{2}-2 A_{1} h_{1}}+e^{N h_{2}-N h_{1}-2 A_{1} h_{2}}\right) \\
& +\left(2 L_{3} N b c_{1}-L_{3} N \xi\right)\left(e^{N h_{2}-N h_{1}-2 A_{1} h_{1}}+e^{N h_{1}-N h_{2}-2 A_{1} h_{2}}\right) \\
& -L_{7} \xi\left(e^{2 N h_{2}-N h_{1}}+e^{2 N h_{1}-N h_{2}}\right)-4 L_{3} N b c_{1}\left(e^{-2 A_{1} h_{1}}+e^{-2 A_{1} h_{2}}\right) \\
& -2 c_{1} N \xi \sinh \left(N h_{1}-N h_{2}\right)+L_{8} N b c_{1}\left(e^{N h_{2}-2 N h_{1}-A_{1} h_{1}}+e^{N h_{1}-2 N h_{2}-A_{1} h_{2}}\right) \\
& \left.+L_{9} N b c_{1}\left(e^{2 N h_{1}-N h_{1}-A_{1} h_{1}}+e^{2 N h_{2}-N h_{1}-A_{1} h_{2}}\right)\right) / D_{7} \text {, }
\end{aligned}
$$




\section{REFERENCES}

1. H. Hottel, A.F. Sarofim, Radiative Transfer, McGraw-Hill Book Company, New York, (1967).

2. B. Jubran, J. Iweida, N. Haimour, Experimental investigation of convective cells generated by side walls in solar ponds using various salts, J. Sol. Energy Eng. 119 (1997) 292-297.

3. K. Petela, G. Manfrida, A. Szlek, Advantages of variable driving temperature in solar absorption chiller, Renew. Energy. 114 (2017) 716-724.

4. M. Padki, S. Sherif, On a simple analytical model for solar chimneys, Int. J. Energy Res. 23 (1999) 345-349.

5. T. Prabu, C.A. Sekar, Improving heat transfer in hybrid solar systems using liquid metal alloys as a coolant, J. Sol. Energy Eng. 139 (2017) 064501.

6. M.M. Rashidi, A. Aghagoli, O. Anwar Bég, Utilization of waste heat in combined power and ejector refrigeration for a solar energy source, Int J Appl Math Mech. 8 (2012) 1-16.

7. C. Zhang, Y. Zhang, X. Lei, W. Gao, Design and comparison of solar thermal oilfield steam production system plans, J. Sol. Energy Eng. 139 (2017) 044502.

8. B.R. Hughes, N.P.S. Cherisa, O. Anwar Bég, Computational study of improving the efficiency of photovoltaic panels in the UAE, WASET J. 73 (2011) 278-287.

9. E. De Schepper, S. Van Passel, S. Lizin, Economic benefits of combining clean energy technologies: the case of solar photovoltaics and battery electric vehicles, Int. J. Energy Res. 39 (2015) 1109-1119.

10. A.K. Tiwari, V.R. Kalamkar, Effects of total head and solar radiation on the performance of solar water pumping system, Renew. Energy. 118 (2018) 919-927.

11. S.U. Choi, J.A. Eastman, Enhancing thermal conductivity of fluids with nanoparticles, Argonne National Lab., IL (United States) (1995).

12. K. Khanafer, K. Vafai, A review on the applications of nanofluids in solar energy field, Renew. Energy. 123 (2018) 398-406.

13. J. Buongiorno, Convective transport in nanofluids, ASME J. Heat Transf. 128 (2006) 240250.

14. R.K. Tiwari, M.K. Das, Heat transfer augmentation in a two-sided lid-driven differentially heated square cavity utilizing nanofluids, Int. J. Heat Mass Transf. 50 (2007) 2002-2018. 
15. R. Shamshirgaran, M.K. Assadi, H. Al-Kayiem, V.S. Korada, Energetic and Exergetic Performance of Solar Flat-Plate Collector Working with Cu Nanofluid, J. Sol. Energy Eng. (2018).

16. A.L. Owolabi, H.H. Al-Kayiem, A.T. Baheta, Performance investigation on a thermal energy storage integrated solar collector system using nanofluid, Int. J. Energy Res. 41 (2017) 650-657.

17. S. Cingarapu, D. Singh, E.V. Timofeeva, M.R. Moravek, Nanofluids with encapsulated tin nanoparticles for advanced heat transfer and thermal energy storage, Int. J. Energy Res. 38 (2014) 51-59.

18. S. Ravindran, Nanofluids in Aerospace Engineering: Solar pumps, rocket propulsion and landing gear tribology, MSc Dissertation, University of Salford, Manchester, UK (2017).

19. M. Abid, T. Ratlamwala, U. Atikol, Performance assessment of parabolic dish and parabolic trough solar thermal power plant using nanofluids and molten salts, Int. J. Energy Res. 40 (2016) 550-563.

20. A. Alashkar, M. Gadalla, Performance analysis of an integrated solar-based power generation plant using nanofluids, Int. J. Energy Res. (2018). DOI: 10.1002/er.3976

21. S. Lim, B. Choi, A study on the MHD (magnetohydrodynamic) micropump with sidewalled electrodes, J. Mech. Sci. Technol. 23 (2009) 739-749.

22. C. Das, G. Wang, F. Payne, Some practical applications of magnetohydrodynamic pumping, Sens. Actuators Phys. 201 (2013) 43-48.

23. J.E. Ho, Characteristic study of MHD pump with channel in rectangular ducts, J. Mar. Sci. Technol. 15 (2007) 315-321.

24. L. Leboucher, P. Boissonneau, D. Villani, Channel shape optimization of electromagnetic pumps, IEEE Trans. Magn. 31 (1995) 2142-2145.

25. P.J. Wang, C.Y. Chang, M.L. Chang, Simulation of two-dimensional fully developed laminar flow for a magneto-hydrodynamic (MHD) pump, Biosens. Bioelectron. 20 (2004) $115-121$.

26. A. Shahidian, M. Ghassemi, R. Mohammadi, Effect of nanofluid properties on magnetohydrodynamic pump (MHD), in: Adv. Mater. Res., Trans Tech Publ, 2012: pp. 663-669. 
27. S. Qian, H.H. Bau, Magneto-hydrodynamics based microfluidics, Mech. Res. Commun. 36 (2009) 10-21.

28. A. Shahidian, M. Ghassemi, S. Khorasanizade, M. Abdollahzade, G. Ahmadi, Flow analysis of non-Newtonian blood in a magnetohydrodynamic pump, IEEE Trans. Magn. 45 (2009) 2667-2670.

29. D. Joo, J. Lee, Numerical study on bubble behavior in magnetic nanofluid used for waste heat recovery power generation concept, Int. J. Energy Res. 42 (2018) 520-531.

30. Novel "magnetohydrodynamics" solar power technology, (n.d.). 28. https://news.rpi.edu/luwakkey/3050.

31. P. Satyamurthy, N. Venkatramani, A. Quraishi, A. Mushtaq, Basic design of a prototype liquid metal magnetohydrodynamic power generator for solar and waste heat, Energy Convers. Manag. 40 (1999) 913-935.

32. S. Kaushik, S. Verma, A. Chandra, Solar-assisted Liquid Metal MHD power generation: A state of the Art Study, Heat Recovery Syst. CHP. 15 (1995) 675-689.

33. M. Romero, J. González-Aguilar, Next generation of liquid metal and other highperformance receiver designs for concentrating solar thermal (CST) central tower systems, in: Adv. Conc. Sol. Therm. Res. Technol., Elsevier, 2017: pp. 129-154.

34. P. Wang, J. Li, K. Vafai, L. Zhao, L. Zhou, Thermo-Fluid Optimization of a Solar Porous Absorber With a Variable Pore Structure, J. Sol. Energy Eng. 139 (2017) 051012.

35. Y. Shi, F. Yin, L. Shi, S. Wence, N. Li, H. Liu, Effects of porous media on thermal and salt diffusion of solar pond, Appl. Energy. 88 (2011) 2445-2453.

36. Y. Ren, H. Qi, J. Shi, Q. Chen, Y. Wang, L. Ruan, Thermal performance characteristics of porous media receiver exposed to concentrated solar radiation, J. Energy Eng. 143 (2017) 04017013.

37. L. Vasiliev, D. Mishkinis, A. Antukh, L. Vasiliev Jr, Solar-gas solid sorption heat pump, Appl. Therm. Eng. 21 (2001) 573-583.

38. M. Al-Nimr, M. Alkam, A modified tubeless solar collector partially filled with porous substrate, Renew. Energy. 13 (1998) 165-173.

39. C.L. Tien, K. Vafai, Convective and radiative heat transfer in porous media, in: Adv. Appl. Mech., Elsevier, 1989: pp. 225-281. 
40. Z. Said, M. Sajid, R. Saidur, M. Kamalisarvestani, N. Rahim, Radiative properties of nanofluids, Int. Commun. Heat Mass Transf. 46 (2013) 74-84.

41. M. Du, G. Tang, Optical property of nanofluids with particle agglomeration, Sol. Energy. 122 (2015) 864-872.

42. O. Anwar Bég, N. Ali, A. Zaman, E.T. Bég, A. Sohail, Computational modeling of heat transfer in an annular porous medium solar energy absorber with the P1-radiative differential approximation, J. Taiwan Inst. Chem. Eng. 66 (2016) 258-268.

43. P. Du, N. Paskaranandavadivel, G. O’Grady, S.J. Tang, L.K. Cheng, A theoretical study of the initiation, maintenance and termination of gastric slow wave re-entry, Math. Med. Biol. J. IMA. 32 (2014) 405-423.

44. P. Slawinski, B. Terry, An automated intestinal biomechanics simulator for expediting robotic capsule endoscope development, J. Med. Devices. 8 (2014) 030901.

45. X. Zheng, Q. Xue, R. Mittal, S. Beilamowicz, A coupled sharp-interface immersed boundary-finite-element method for flow-structure interaction with application to human phonation, ASME J. Biomech. Eng. 132 (2010) 111003.

46. R. Schulz, A.V. Vargiu, F. Collu, U. Kleinekathöfer, P. Ruggerone, Functional rotation of the transporter Acrb: the essentials of peristaltic motion and subsequent substrate extrusion, Biophys. J. 98 (2010) 685a.

47. A. Pal, J.G. Brasseur, The mechanical advantage of local longitudinal shortening on peristaltic transport, ASME J. Biomech. Eng. 124 (2002) 94-100.

48. Y.Y. Tsui, D.C. Guo, S.-H. Chen, S.-W. Lin, Pumping flow in a channel with a peristaltic wall, ASME J. Fluids Eng. 136 (2014) 021202.

49. A. Dobrolyubov, G. Douchy, Peristaltic transport as the travelling deformation waves, $J$. Theor. Biol. 219 (2002) 55-61.

50. Y.R. Kumar, M.R. Murthy, S. Sreenadh, others, Unsteady peristaltic pumping in a finite length tube with permeable wall, ASME J. Fluids Eng. 132 (2010) 101201.

51. H. Moradi, S. Zandi, J. Floryan, Algorithm for analysis of peristaltic annular flows, Comput. Fluids. 147 (2017) 72-89.

52. M. Reddy, M. Mishra, S. Sreenadh, A.R. Rao, Influence of lateral walls on peristaltic flow in a rectangular duct, ASME J. Fluids Eng. 127 (2005) 824-827. 
53. X. Mandviwalla, R. Archer, The influence of slip boundary conditions on peristaltic pumping in a rectangular channel, ASME J. Fluids Eng. 130 (2008) 124501.

54. K. Ramesh, Influence of heat and mass transfer on peristaltic flow of a couple stress fluid through porous medium in the presence of inclined magnetic field in an inclined asymmetric channel, J. Mol. Liq. 219 (2016) 256-271.

55. D. Tripathi, O. Anwar Bég, A study of unsteady physiological magneto-fluid flow and heat transfer through a finite length channel by peristaltic pumping, Proc. Inst. Mech. Eng. Part H. J Eng. Medicine. 226 (2012) 631-644.

56. O. Anwar Bég, D. Tripathi, Mathematica simulation of peristaltic pumping with doublediffusive convection in nanofluids: a bio-nano-engineering model, Proc. Inst. Mech. Eng. Part N J. Nanoeng. Nanosyst. 225 (2011) 99-114.

57. M.M. Bhatti, A. Zeeshan, R. Ellahi, Endoscope analysis on peristaltic blood flow of Sisko fluid with Titanium magneto-nanoparticles, Comput. Biol. Med. 78 (2016) 29-41.

58. N.S. Akbar, D. Tripathi, O. Anwar Bég, Modeling nanoparticle geometry effects on peristaltic pumping of medical magnetohydrodynamic nanofluids with heat transfer, $J$. Mech. Med. Biol. 16 (2016) 1650088.

59. D. Tripathi, R. Jhorar, O. Anwar Bég, A. Kadir, Electro-magneto-hydrodynamic peristaltic pumping of couple stress biofluids through a complex wavy micro-channel, J. Mol. Liq. 236 (2017) 358-367.

60. M. Bhatti, A. Zeeshan, N. Ijaz, O. Anwar Bég, A. Kadir, Mathematical modelling of nonlinear thermal radiation effects on EMHD peristaltic pumping of viscoelastic dusty fluid through a porous medium duct, Eng. Sci. Technol. Int. J. 20 (2017) 1129-1139.

61. T. Hayat, S. Rani, A. Alsaedi, M. Rafiq, Radiative peristaltic flow of magneto nanofluid in a porous channel with thermal radiation, Results Phys. 7 (2017) 3396-3407.

62. S. Nadeem, and N. S. Akbar, Effects of heat transfer on the peristaltic transport of MHD Newtonian fluid with variable viscosity: application of Adomian decomposition method. Commun. Nonlinear Sci. Numer. Simul. 14 (2009) 3844-3855.

63. M.J. Uddin, W. Khan, A.M. Ismail, O. Anwar Bég, Computational study of threedimensional stagnation point nanofluid bioconvection flow on a moving surface with anisotropic slip and thermal jump effect, ASME J. Heat Transfer. 138 (2016) 104502.

64. S. Srinivas, M. Kothandapani, Peristaltic transport in an asymmetric channel with heat transfer - A note, Int. J. Heat Mass Transf. 35 (2008) 514-522. 